\title{
Supernovae and Weinberg's Higgs portal dark radiation and dark matter
}

\author{
Huitzu Tu and Kin-Wang Ng \\ Institute of Physics, Academia Sinica, \\ 128 Academia Road, Section 2, Nankang, Taipei 11529, Taiwan \\ E-mail: huitzu2@gate.sinica.edu.tw, nkw@phys.sinica.edu.tw
}

ABSTRACT: The observed burst duration and energies of the neutrinos from Supernova 1987A strongly limit the possibility of any weakly-interacting light particle species being produced in the proto-neutron star (PNS) core and leading to efficient energy loss. We reexamine this constraint on Weinberg's Higgs portal model, in which the dark radiation particles (the Goldstone bosons) and the dark matter candidate (a Majorana fermion) interact with Standard Model (SM) fields solely through the mixing of the SM Higgs boson and a light Higgs boson. In order for the Goldstone bosons to freely stream out of the PNS core region, the Higgs portal coupling has to be about a factor of 4-9 smaller than the current collider bound inferred from the SM Higgs invisible decay width. We find that in the energy loss rate calculations, results obtained by using the one-pion exchange (OPE) approximation and the SP07 global fits for the nucleon-nucleon total elastic cross section differ only by a factor $\lesssim 3$. The SN 1987A constraints surpass those set by laboratory experiments or by the energy loss arguments in other astrophysical objects such as the gamma-ray bursts, even with other nuclear uncertainties taken into account. Furthermore, the SN 1987A constraints are comparable to bounds from the latest dark matter direct search for low-mass WIMPs $(\lesssim 10 \mathrm{GeV}$.)

KeYwords: Cosmology of Theories beyond the SM, Higgs Physics

ARXIV EPRINT: 1706.08340 


\section{Contents}

1 Introduction 1

2 Weinberg's Higgs portal model 2

3 Goldstone boson production in proto-neutron star core 4

3.1 Energy loss rate using one-pion exchange approximation 5

$\begin{array}{lll}3.1 .1 & \text { Non-degenerate limit } & 7\end{array}$

3.1.2 Degenerate limit 8

$\begin{array}{lll}3.2 & \text { Energy loss rate using phase shifts data } & 10\end{array}$

$\begin{array}{lll}\text { 3.2.1 Global fits of total elastic cross sections } & 11\end{array}$

$\begin{array}{lll}\text { 3.2.2 Chiral effective field theory predictions } & 13\end{array}$

4 Goldstone boson propagation in proto-neutron star core 15

5 SN 1987A constraints on Weinberg's Higgs portal model 17

$\begin{array}{lll}6 & \text { Summary } & 21\end{array}$

\section{Introduction}

SN 1987A was a type II supernova discovered on February 24, 1987 by Shelton, Duhalde and Jones. The progenitor star was Sanduleak $-69^{\circ} 202$, a blue supergiant in the Large Magellanic Cloud. Thanks to its proximity of about $51 \mathrm{kpc}$ to the Earth, neutrino burst events from the core collapse of the progenitor star could be recorded at the underground laboratories Irvine-Michigan-Brookhaven (IMB), Kamiokande II, and Baksan separately [1]. The observed burst duration of about 12 seconds, individual energies up to $40 \mathrm{MeV}$, as well as the integrated total energy of $\mathcal{O}\left(10^{53} \mathrm{erg}\right)$, confirmed the standard picture of neutrino cooling of the proto-neutron star (PNS) [2-4]. A proto-neutron star is formed when the collapsing stellar core of the progenitor star reaches nuclear saturation density. Being initially hot and lepton rich, the PNS keeps contracting as it cools and deleptonises, to become a neutron star as the final supernova remnant. See refs. [5-7] for the PNS structure and the evolution, and ref. [8] for the most recent review on neutrino emission from supernovae.

Emission of light exotic particles in nuclear interactions in the PNS core have been considered exhaustively in the literature, notably the axions [9-13], right-handed neutrinos [9], Kaluza-Klein gravitons [14-16], Kaluza-Klein dilatons [14], unparticles [17, 18], dark photons [19, 20], dark matter [21], dilation [22], saxion [23] etc. Simulations of PNS in the neutrino-emitting phase were done in refs. [24, 25] for the axion, and in ref. [15] for the KK-gravitons. By comparing the predicted neutrino burst signals with the SN 1987A observations, very stringent constraints were obtained on the properties of the exotic particles. For a quick comparison without invoking simulations, Raffelt has derived a bound on the emissivity of light exotic particles based on the argument that they should not affect the total cooling time significantly [26, 27]. 
In this work we shall reexamine the SN 1987A constraints on Weinberg's Higgs portal model [28], which was proposed to account for the dark radiation in the early universe. The effect of the dark radiation on the cosmic microwave background (CMB) data is parametrised as the contribution to the effective number of light neutrino species $N_{\text {eff }}$. The conflict between the value of the Hubble constant $H_{0}$ from the Planck CMB data and local determination may be remedied by assuming an addition of $\Delta N_{\text {eff }}=0.4-1$ to the standard value of $N_{\nu}=3.046$ by the dark radiation component [29] (see, however, also ref. [30].) In this model, Weinberg considered a global U(1) continuous symmetry associated with the conservation of some quantum number, and introduced a complex scalar field to break it spontaneously. The radial field of the complex scalar field acquires a vacuum expectation value (vev), and mixes with the Standard Model (SM) Higgs field. The Goldstone bosons arising from the symmetry breaking would be massless, and their characteristic derivative coupling would make them very weakly-interacting at sufficiently low temperatures. The latter property is crucial, since the Goldstone bosons must decouple from the early universe thermal bath at the right moment so that their temperature is a fraction of that of the neutrinos (see e.g. ref. [31].) Collider phenomenology of Weinberg's Higgs portal model has been investigated in refs. $[32,33]$. Weinberg has also extended this minimal set-up to include a Majorana fermion as a Weakly-Interacting Massive Particle (WIMP) dark matter candidate. In ref. [33] it was shown that the results of the dark matter direct search experiments LUX [34] provide very strong constraints, which are slightly strengthened by the XENON1T experiment [35] very recently.

Previously we have examined energy losses due to the emission of Weinberg's Goldstone bosons in a post-collapse supernova core [36] in the limit of large radial field mass. Subsequently we scrutinised the production and propagation of Weinberg's Goldstone bosons in the initial fireballs of gamma-ray bursts for more general cases [37]. In this work we extend our previous analysis and consider in greater detail Goldstone boson production by nuclear bremsstrahlung processes in the proto-neutron star core of SN 1987A. In section 2 we briefly review Weinberg's Higgs portal model for dark radiation and dark matter. In section 3 we calculate energy loss rate due to Goldstone boson emission by two methods, i.e. using the one-pion exchange approximation and using experimental data of low-energy nucleon collisions. In section 4 we estimate the mean free path of the Goldstone bosons as a function of their emission energies, and determine the free-streaming requirements. Our results in these two sections are then used in section 5 to derive supernova constraints on Weinberg's Higgs portal model by invoking Raffelt's criterion. We then confront our SN 1987A constraints with those from accelerator experiments, gamma-ray burst observations, and dark matter direct search experiments. In section 6 we summarise our work.

\section{Weinberg's Higgs portal model}

In this section we briefly summarise Weinberg's model [28] following the convention of refs. $[32,36]$. Consider the simplest possible broken continuous symmetry, a global U(1) symmetry associated with the conservation of some quantum number $W$. A single complex scalar field $S(x)$ is introduced for breaking this symmetry spontaneously. With this field 
added to the Standard Model (SM), the Lagrangian is

$$
\mathcal{L}=\left(\partial_{\mu} S^{\dagger}\right)\left(\partial^{\mu} S\right)+\mu^{2} S^{\dagger} S-\lambda\left(S^{\dagger} S\right)^{2}-g\left(S^{\dagger} S\right)\left(\Phi^{\dagger} \Phi\right)+\mathcal{L}_{\mathrm{SM}},
$$

where $\Phi$ is the SM Higgs doublet, $\mu^{2}, g$, and $\lambda$ are real constants, and $\mathcal{L}_{\mathrm{SM}}$ is the usual SM Lagrangian. One separates a massless Goldstone boson field $\alpha(x)$ and a massive radial field $r(x)$ in $S(x)$ by defining

$$
S(x)=\frac{1}{\sqrt{2}}(\langle r\rangle+r(x)) e^{2 i \alpha(x)},
$$

where the fields $\alpha(x)$ and $r(x)$ are real. In the unitary gauge, one sets $\Phi^{\mathrm{T}}=(0,\langle\varphi\rangle+\varphi(x)) / \sqrt{2}$ where $\varphi(x)$ is the physical Higgs field. The Lagrangian in eq. (2.1) thus becomes

$$
\begin{aligned}
\mathcal{L}= & \frac{1}{2}\left(\partial_{\mu} r\right)\left(\partial^{\mu} r\right)+\frac{1}{2} \frac{(\langle r\rangle+r)^{2}}{\langle r\rangle^{2}}\left(\partial_{\mu} \alpha\right)\left(\partial^{\mu} \alpha\right)+\frac{\mu^{2}}{2}(\langle r\rangle+r)^{2} \\
& -\frac{\lambda}{4}(\langle r\rangle+r)^{4}-\frac{g}{4}(\langle r\rangle+r)^{2}(\langle\varphi\rangle+\varphi)^{2}+\mathcal{L}_{\mathrm{SM}},
\end{aligned}
$$

where the replacement $\alpha(x) \rightarrow \alpha(x) /(2\langle r\rangle)$ was made in order to achieve a canonical kinetic term for the $\alpha(x)$ field. The two fields $\varphi$ and $r$ mix due to the $g\left(S^{\dagger} S\right)\left(\Phi^{\dagger} \Phi\right)$ term, with their mixing angle given by

$$
\tan 2 \theta=\frac{2 g\langle\varphi\rangle\langle r\rangle}{m_{H}^{2}-m_{h}^{2}}
$$

where $m_{H}$ and $m_{h}$ are the masses of the two resulting physical Higgs bosons $H$ and $h$, respectively. The heavier one is identified with the SM Higgs boson with $m_{H}=125 \mathrm{GeV}$, while the lighter one is assumed to have a mass in the range of $\mathrm{MeV}$ to hundreds of $\mathrm{MeV}$. In this model, the interaction of the Goldstone bosons with the SM fields arises entirely through the SM Higgs boson in the mixing of the $\varphi$ and $r$ fields. The light Higgs boson $h$ decays dominantly to a pair of Goldstone bosons, with the decay width given by

$$
\Gamma_{h}=\frac{1}{32 \pi} \frac{m_{h}^{3}}{\langle r\rangle^{2}}
$$

When kinematically allowed, there is also a probability for $h$ decaying into a pair of SM fermions as well as a pair of pions [37].

The Higgs effective coupling to nucleons, $f_{N} m_{N} /\langle\varphi\rangle \equiv g_{N N H}$, has been calculated for the purpose of investigating the sensitivities of the dark matter direct detection experiments [38-42]. For example, ref. [41] found $g_{N N H}=0.0011$, which corresponds to $f_{N} \simeq 0.288$. It was pointed out in ref. [43] that the effective Higgs-nucleon coupling has a wide range of values, $0.0011 \leq g_{N N H} \leq 0.0032$, due to uncertainties in the pion-nucleon sigma term. The authors of ref. [42] have done a statistical analysis to infer the value of $f_{N}$ from more up-to-date lattice evaluations of the nucleon matrix elements. By exploiting two possible statistical distributions for the strangeness matrix element, they found $f_{N}=0.3 \pm 0.03$ and $f_{N}=0.3 \pm 0.01$ at the $68 \%$ confidence level, respectively. 
This model is also extended to include a dark matter candidate by adding one Dirac field

$$
\mathcal{L}_{\psi}=i \bar{\psi} \gamma \cdot \partial \psi-m_{\psi} \bar{\psi} \psi-\frac{f_{\chi}}{\sqrt{2}} \bar{\psi}^{c} \psi S^{\dagger}-\frac{f_{\chi}^{*}}{\sqrt{2}} \bar{\psi} \psi^{c} S,
$$

and assigning a charge $\mathrm{U}(1)_{W}=1$ for it. One expresses the field as $\psi(x)=\psi^{\prime}(x) e^{i \alpha(x)}$, and expands the Lagrangian after the radial field achieves a vev (for details see ref. [33].) Diagonalising the $\psi^{\prime}$ mass matrix generates the mass eigenvalues

$$
m_{ \pm}=m_{\psi} \pm f_{\chi}\langle r\rangle
$$

for the two mass eigenstates $\psi_{ \pm}$, which are Majorana fermions. The Lagrangian is now

$$
\begin{aligned}
\mathcal{L}_{\psi}= & \frac{i}{2} \bar{\psi}_{ \pm} \gamma \cdot \partial \psi_{ \pm}-\frac{1}{2} m_{ \pm} \bar{\psi}_{ \pm} \psi_{ \pm}-\frac{i}{4\langle r\rangle}\left(\bar{\psi}_{+} \gamma \psi_{-}-\psi \psi_{-} \gamma \psi_{+}\right) \cdot \partial \alpha \\
& -\frac{f_{\chi}}{2} r\left(\bar{\psi}_{+} \psi_{+}-\bar{\psi}_{-} \psi_{-}\right),
\end{aligned}
$$

and one needs to use the massive representation $r=\cos \theta h+\sin \theta H$ for the interactions of $\psi_{ \pm}$. The heavier fermion decays into the lighter fermion by emitting a Goldstone boson, while the lighter one is stable due to unbroken reflection symmetry. The latter can thus play the role of the WIMP dark matter, with mass $m_{-} \equiv M_{\chi}$ in the range of $\mathrm{GeV}$ to $\mathrm{TeV}$. Its relic density has been calculated in ref. [44].

Model parameters in the minimal set-up are $m_{h}, g$, and $\langle r\rangle$, and including $M_{\chi}$ and $f_{\chi}$ in the extended version. From the SM Higgs invisible decay width, a collider bound on the Higgs portal coupling

$$
g<0.011,
$$

has been derived in ref. [32]. In the future, the International Linear Collider (ILC) may reach a sensitivity of constraining the branching ratio of SM Higgs invisible decays to $<0.4-0.9 \%$ [45] in the best scenarios. If this can be realised, the collider bound on the Goldstone boson coupling will be improved by a factor of $5 \sim 7$. Experimental limits on meson invisible decay widths have also been turned into constraints on the $\varphi-r$ mixing angle in ref. [33], which we list in section 5. There is also the perturbativity condition, which requires for the quartic self-coupling of the $S$ field

$$
\lambda=\frac{m_{h}^{2}}{\langle r\rangle^{2}} \leq 4 \pi .
$$

In Weinberg's Higgs portal model including the dark matter candidate, exclusion limits on the WIMP-nucleon elastic cross section set by the null results of the direct search experiments have been found to put very strong bounds on the mixing angle in ref. [33].

\section{Goldstone boson production in proto-neutron star core}

In the PNS core, the dominant Goldstone boson production channel is the nuclear bremsstrahlung processes $N N \rightarrow N N \alpha \alpha$. Low-energy nuclear interactions have been studied quite thoroughly by various experiments, while theoretical calculation remains a difficult 
task. Taketani, Nakamura and Sasaki [46] suggested to divide the nuclear forces into three regions: the classical (long-range), a dynamical (intermediate range), and a phenomenological or core (short-range) region. In the classical region, the one-pion exchange (OPE) dominates the longest range part of the potential. In the intermediate range the two-pion exchange (TPE) is most important, where heavier mesons may also become relevant. In the short-range region, multi-pion exchange, heavy mesons, quark-gluon exchanges are expected to be responsible. At present $N N$ potentials calculated using the chiral effective field theory to the fifth order $\left(\mathrm{N}^{4} \mathrm{LO}\right)$ [47] and the sixth order $\left(\mathrm{N}^{5} \mathrm{LO}\right)$ [48] are available, which can reproduce the experimental data to outstanding precision. See e.g. refs. [49-52] for reviews on nucleon-nucleon interactions.

As for nuclear bremsstrahlung processes, in refs. $[53,54]$ neutrino pair production in core-collapse supernovae was studied using chiral effective field theory to the fourth order $\left(\mathrm{N}^{3} \mathrm{LO}\right)$. It was found that shorter-range noncentral forces significantly reduce the neutrino rates compared to the one-pion exchange (OPE) approximation $[12,55,56]$, which was typically used in supernova simulations or in deriving supernova bounds on exotic particles. More recently, ref. [57] goes beyond the OPE approach and uses $T$-matrix based formalism from ref. [58] in their supernova simulations. The approach of using phase shift data to fix the on-shell $N N$ scattering amplitudes and making the soft-radiation approximation has already been taken in ref. [59] much earlier. It was found therein that the resultant rates are roughly a factor of four below earlier estimates based on an OPE $N N$ amplitude.

In this section we make the same comparison in Weinberg's Higgs portal model.

\subsection{Energy loss rate using one-pion exchange approximation}

The OPE contribution to the nuclear forces takes care of the long-range interactions and the tensor force. From the Lagrangian describing the pion coupling to nucleons $\mathcal{L}_{\pi^{0} N N}=-g_{\pi^{0}} \bar{\psi} i \gamma^{5} \tau_{3} \psi \varphi^{\left(\pi^{0}\right)}$, where $N=n, p$, the potential is

$$
V_{\mathrm{OPE}}(\vec{k})=-\left(\frac{f_{\pi}}{m_{\pi}}\right)^{2} \frac{\left(\vec{\sigma}_{1} \cdot \vec{k}\right)\left(\vec{\sigma}_{2} \cdot \vec{k}\right)}{|\vec{k}|^{2}+m_{\pi}^{2}}\left(\vec{\tau}_{1} \cdot \vec{\tau}_{2}\right),
$$

with $\vec{k}$ the momentum exchange, and $\vec{\sigma}_{j}$ and $\vec{\tau}_{i}$ the spin and isospin operators of the incoming nucleons, respectively. The neutral pion-nucleon coupling constant is $g_{\pi^{0}}^{2} / 4 \pi=$ $\left(2 m_{N} f_{\pi} / m_{\pi}\right)^{2} /(4 \pi) \approx 14[60,61]$, with $f_{\pi} \approx 1$. In the one-pion exchange (OPE) approximation (see e.g. ref. [12]), there are four direct and four exchange diagrams, corresponding to the Goldstone boson pairs being emitted by any one of the nucleons. Summing all diagrams and expanding in powers of $\left(T / m_{N}\right)$, the amplitude for the nuclear bremsstrahlung processes $N\left(p_{1}\right) N\left(p_{2}\right) \rightarrow N\left(p_{3}\right) N\left(p_{4}\right) \alpha\left(q_{1}\right) \alpha\left(q_{2}\right)$ is [36]

$$
\begin{aligned}
\sum_{\text {spins }} & \left|\mathcal{M}_{N N \rightarrow N N \alpha \alpha}^{\mathrm{OPE}}\right|^{2} \approx 64\left(\frac{f_{N} g m_{N}}{m_{H}^{2}}\right)^{2}\left(\frac{2 m_{N} f_{\pi}}{m_{\pi}}\right)^{4} \frac{\left(q_{1} \cdot q_{2}\right)^{2}}{\left(q^{2}-m_{h}^{2}\right)^{2}+m_{h}^{2} \Gamma_{h}^{2}} \\
& \times \frac{\left(-2 q^{2}\right)^{2} m_{N}^{2}}{(2 p \cdot q)^{4}}\left\{\frac{|\vec{k}|^{4}}{\left(|\vec{k}|^{2}+m_{\pi}^{2}\right)^{2}}+\frac{|\vec{l}|^{4}}{\left(|\vec{l}|^{2}+m_{\pi}^{2}\right)^{2}}+\frac{|\vec{k}|^{2}|\vec{l}|^{2}-2|\vec{k} \cdot \vec{l}|^{2}}{\left(|\vec{k}|^{2}+m_{\pi}^{2}\right)\left(|\vec{l}|^{2}+m_{\pi}^{2}\right)}+\ldots\right\},
\end{aligned}
$$


where $q \equiv q_{1}+q_{2}$, and $k \equiv p_{2}-p_{4}$ and $l \equiv p_{2}-p_{3}$ are the 4-momenta of the exchanged pion in the direct and the exchange diagrams, respectively. In addition, Goldstone boson pairs can be emitted from the exchanged pion due to an effective Higgs-pion coupling. The amplitude for this process is

$$
\begin{aligned}
\sum_{\text {spins }}\left|\mathcal{M}_{N N \rightarrow N N \alpha \alpha}^{\text {OPE (pion) }}\right|^{2} \approx & 4\left(\frac{g}{m_{H}^{2}}\right)^{2}\left(\frac{2 m_{N} f_{\pi}}{m_{\pi}}\right)^{4} \frac{\left(q_{1} \cdot q_{2}\right)^{2}}{\left(q^{2}-m_{h}^{2}\right)^{2}+m_{h}^{2} \Gamma_{h}^{2}}\left(\frac{2}{9}\right)^{2} \\
& \times\left(q^{2}+\frac{11}{2} m_{\pi}^{2}\right)^{2}\left\{\frac{k_{1}^{2} k_{2}^{2}}{\left(k_{1}^{2}-m_{\pi}^{2}\right)^{2}\left(k_{2}^{2}-m_{\pi}^{2}\right)^{2}}+\frac{l_{1}^{2} l_{2}^{2}}{\left(l_{1}^{2}-m_{\pi}^{2}\right)^{2}\left(l_{2}^{2}-m_{\pi}^{2}\right)^{2}}\right. \\
& \left.+\frac{\left(k_{1} \cdot k_{2}\right)\left(l_{1} \cdot l_{2}\right)+\ldots}{\left(k_{1}^{2}-m_{\pi}^{2}\right)\left(k_{2}^{2}-m_{\pi}^{2}\right)\left(l_{1}^{2}-m_{\pi}^{2}\right)\left(l_{2}^{2}-m_{\pi}^{2}\right)}\right\},
\end{aligned}
$$

where $k_{1} \equiv p_{1}-p_{3}, k_{2} \equiv p_{2}-p_{4}, l_{1} \equiv p_{1}-p_{4}$, and $l_{2} \equiv p_{2}-p_{3}$, with $k_{1}+k_{2}=l_{1}+l_{2}=q$. However, with $q^{2} \approx m_{h}^{2}, k_{1}^{2} \simeq-|\vec{k}|^{2}$ and similarly for $k_{2}^{2}, l_{1}^{2}$, and $l_{2}^{2}$, this contribution is subdominant.

The volume energy loss rate is

$$
\begin{aligned}
Q_{N N \rightarrow N N \alpha \alpha}= & \frac{\mathcal{S}}{2 !} \int \frac{d^{3} \overrightarrow{q_{1}}}{2 \omega_{1}(2 \pi)^{3}} \frac{d^{3} \overrightarrow{q_{2}}}{2 \omega_{2}(2 \pi)^{3}} \int \prod_{i=1}^{4} \frac{d^{3} \overrightarrow{p_{i}}}{2 E_{i}(2 \pi)^{3}} f_{1} f_{2}\left(1-f_{3}\right)\left(1-f_{4}\right) \\
& \times \sum_{\text {spins }}\left|\mathcal{M}_{N N \rightarrow N N \alpha \alpha}\right|^{2}(2 \pi)^{4} \delta^{4}\left(p_{1}+p_{2}-p_{3}-p_{4}-q_{1}-q_{2}\right)\left(\omega_{1}+\omega_{2}\right),
\end{aligned}
$$

where $\omega_{1}, \omega_{2}$ are the energy of the Goldstone bosons in the final state. The symmetry factor $\mathcal{S}$ is $\frac{1}{4}$ for $n n$ and $p p$ interactions, whereas for $n p$ interactions it is 1 . The nucleon occupation numbers are $f_{i}=1 /\left(e^{\left(E_{i}-\mu_{N}\right) / T}+1\right)$, where in the non-relativistic limit the nucleon energies are

$$
E_{i} \simeq m_{N}+\frac{\left|\vec{p}_{i}\right|^{2}}{2 m_{N}}+U_{N} .
$$

Here $\mu_{N}$ is the chemical potential of the nucleon, and $U_{N}$ is the mean-field single-particle potential in which the nucleons move. In ref. [62] it is pointed out that due to the extreme neutron-rich conditions in the PNS core, the mean-field potentials for neutrons and protons can differ significantly, with the difference directly related to the nuclear symmetry energy (see e.g. refs. $[63,64]$ for recent reviews.) Non-zero $U_{n}-U_{p}$ was found therein to have a strong impact on the spectra and luminosities of the supernova emitted neutrinos. In any case the nucleon occupation numbers are normalised to the nucleon number density,

$$
n_{N}=X_{N} n_{B}=\int_{0}^{\infty} \frac{2 d^{3} \vec{p}_{i}}{(2 \pi)^{3}} f_{i}\left(\vec{p}_{i}\right)
$$

where $n_{B}$ is the total baryon number density, and $X_{N}$ with $N=n, p$, are the neutron and the proton fraction, respectively. The relative abundances of the neutrons, protons, electrons, and the neutrinos in the PNS core are determined by the conditions of kinetic and chemical equilibrium, as well as charge neutrality. Therefore the neutron fraction $X_{n}$ parametrises the underlying nuclear equation of state and indicates the level of neutron degeneracy. 
We perform the integral over the Goldstone boson momenta first

$$
\int \frac{d^{3} \overrightarrow{q_{1}}}{\omega_{1}} \frac{d^{3} \overrightarrow{q_{2}}}{\omega_{2}} \frac{\left(q_{1} \cdot q_{2}\right)^{2}}{\left(q^{2}-m_{h}^{2}\right)^{2}+m_{h}^{2} \Gamma_{h}^{2}} \frac{\left(2 q^{2}\right)^{2}}{(2 p \cdot q)^{4}} \omega=\frac{2(2 \pi)^{2}}{m_{N}^{4}} \int_{0}^{\infty} d \omega \omega^{4} I_{1}\left(\omega, m_{h},\langle r\rangle\right),
$$

where $\omega=\omega_{1}+\omega_{2}$. The dimensionless integral is defined by

$$
I_{1}\left(\omega, m_{h},\langle r\rangle\right) \equiv \int_{0}^{1} d \tilde{\omega} \int_{-1}^{+1} \frac{d \cos \theta \tilde{\omega}^{5}(1-\tilde{\omega})^{5}(1-\cos \theta)^{4}}{\left[2 \tilde{\omega}(1-\tilde{\omega})(1-\cos \theta)-\frac{m_{h}^{2}}{\omega^{2}}\right]^{2}+\frac{m_{h}^{2} \Gamma_{h}^{2}}{\omega^{4}}},
$$

with $\tilde{\omega} \equiv \omega_{1} / \omega$, and $\theta$ is the angle between the two emitted Goldstone bosons.

As the integral over the nucleon momenta in eq. (3.4) is not easy to evaluate, we follow the conventional approach of taking the non-degenerate and the degenerate limit in the following. As we will show, energy loss rate due to Goldstone boson emission calculated in these two limits have distinct dependences on the PNS core temperature $T$ and neutron fraction $X_{n}$ therein.

\subsubsection{Non-degenerate limit}

The initial-state nucleon occupation numbers are given by the non-relativistic MaxwellBoltzmann distribution $f_{i}\left(\vec{p}_{i}\right)=\left(n_{N} / 2\right)\left(2 \pi / m_{N} T\right)^{3 / 2} e^{-\left|\vec{p}_{i}\right|^{2} / 2 m_{N} T}$. The integration is simplified by introducing the center-of-mass momenta, so that $\vec{p}_{1,2}=\vec{P} \pm \vec{p}_{i}$, and $\vec{p}_{3,4}=\vec{P} \pm \vec{p}_{f}$. The $d^{3} \vec{P}$ integral can be performed separately. The energy loss rate in the non-degenerate limit is then

$$
Q_{N N \rightarrow N N \alpha \alpha}^{\mathrm{OPE}(\mathrm{ND})}=\frac{\mathcal{S} \sqrt{\pi}}{(2 \pi)^{6}}\left(3-\frac{2 \beta}{3}\right) I_{0} n_{N}^{2}\left(\frac{f_{N} g m_{N}}{m_{H}^{2}}\right)^{2}\left(\frac{2 m_{N} f_{\pi}}{m_{\pi}}\right)^{4} \cdot \frac{T^{5.5}}{m_{N}^{4.5}} .
$$

Here we have defined the integral $I_{0}$ by

$$
I_{0}\left(T, m_{h},\langle r\rangle\right) \equiv \int d u d v d x x^{4} I_{1}\left(x, T, m_{h},\langle r\rangle\right) \sqrt{u v} e^{-u} \delta(u-v-x),
$$

with $u \equiv\left|\vec{p}_{i}\right|^{2} / m_{N} T, v \equiv\left|\vec{p}_{f}\right|^{2} / m_{N} T$, and $x \equiv \omega / T$. The $\beta$ term is

$$
\beta \equiv \frac{3}{I_{0}} \int d u d v d x x^{4} I_{1}\left(x, m_{h},\langle r\rangle\right) \sqrt{u v} e^{-u} \delta(u-v-x) \int_{-1}^{+1} \frac{d z}{2} \frac{|\vec{k} \cdot \vec{l}|^{2}}{|\vec{k}|^{2}|\vec{l}|^{2}},
$$

where $z \equiv\left(\vec{p}_{i} \cdot \vec{p}_{f}\right) /\left|\vec{p}_{i}\right|\left|\vec{p}_{f}\right|$, the angle between $\vec{p}_{i}$ and $\vec{p}_{f}$.

In the resonance region, one can make use of the limit of the Poisson kernel

$$
\lim _{\epsilon \rightarrow 0} \frac{1}{\pi} \frac{\epsilon}{a^{2}+\epsilon^{2}}=\delta(a)
$$

and obtain

$$
I_{1}^{\mathrm{Pk}}\left(\omega, m_{h},\langle r\rangle\right) \approx \frac{\pi}{32} \frac{m_{h}^{7}}{\Gamma_{h} \omega^{6}} .
$$

Since this approximation is valid when $m_{h}^{2} / \omega^{2} \approx 2 \tilde{\omega}(1-\tilde{\omega})$, where the latter $\leq 1$, it is only applicable for $\omega \geq m_{h}$ and $\Gamma_{h} \ll \omega$. We have checked that, for $m_{h}=500 \mathrm{MeV}$ and $\langle r\rangle=10 \mathrm{GeV}$, this approximation still works well. 
This is equivalent to considering the production of a real light Higgs boson $h$, for which

$$
\begin{aligned}
Q_{N N \rightarrow N N h}^{\mathrm{OPE}(\mathrm{ND})}= & \frac{\mathcal{S} \sqrt{\pi}}{4(2 \pi)^{4}}\left(3-\frac{2 \beta}{3}\right) n_{N}^{2}\left(\frac{f_{N} g\langle r\rangle m_{N}}{m_{H}^{2}}\right)^{2}\left(\frac{2 m_{N} f_{\pi}}{m_{\pi}}\right)^{4} \frac{m_{h}^{4}}{m_{N}^{9 / 2} T^{1 / 2}} \\
& \times \int_{m_{h} / T}^{\infty} d x \frac{\sqrt{x^{2}-\frac{m_{h}^{2}}{T^{2}}}}{x^{3}} \int_{0}^{\infty} d u d v \sqrt{u v} e^{-u} \delta(u-v-x) .
\end{aligned}
$$

And indeed we find that for $m_{h} \lesssim 500 \mathrm{MeV}$,

$$
Q_{N N \rightarrow N N \alpha \alpha}^{\mathrm{Pk}} \approx Q_{N N \rightarrow N N h} \times \mathcal{B}(h \rightarrow \alpha \alpha),
$$

with $\mathcal{B}(h \rightarrow \alpha \alpha)=\Gamma_{h \rightarrow \alpha \alpha} / \Gamma_{h}$ the branching ratio of the light Higgs boson $h$ decaying into a pair of Goldstone bosons. Thus we find that in the parameter range we consider in this work, Goldstone boson production in the PNS core is dominated by the production of a real light Higgs boson $h$ and its subsequent decay. This is a very distinct feature from the nuclear bremsstrahlung emission of a massless scalar, e.g. the dilaton [22], or a massive stable scalar such as the saxion [23].

\subsubsection{Degenerate limit}

We calculate the energy loss rate due to Goldstone boson emission, eq. (3.4), in the degenerate limit following ref. [55]. The integral over the Goldstone boson momenta is done as in eq. (3.7) and eq. (3.8) first. In the degenerate limit, the nucleon momenta integral is simplified by $d^{3} \vec{p}_{i}=\left|\vec{p}_{i}\right|^{2} d\left|\vec{p}_{i}\right| d \Omega_{i} \approx p_{F}(n) m_{N} d E_{i} d \Omega_{i}$. The neutron Fermi momentum is $p_{F}(n)=\left(3 \pi^{2} n_{n}\right)^{1 / 3}$, with the neutron number density $n_{n}=X_{n} \rho / m_{N}$ given by eq. (3.6). One then perform the integral

$$
\begin{aligned}
\left\langle F_{N N}\right\rangle \equiv & \frac{(4 \pi)^{2}}{A} \int \prod_{i=1}^{4} d \Omega_{i} \delta^{3}\left(\vec{p}_{1}+\vec{p}_{2}-\vec{p}_{3}-\vec{p}_{4}\right) \\
& \times\left\{\frac{|\vec{k}|^{4}}{\left(|\vec{k}|^{2}+m_{\pi}^{2}\right)^{2}}+\frac{|\vec{l}|^{4}}{\left(|\vec{l}|^{2}+m_{\pi}^{2}\right)^{2}}+\frac{|\vec{k}|^{2}|\vec{l}|^{2}-2|\vec{k} \cdot \vec{l}|^{2}}{\left(|\vec{k}|^{2}+m_{\pi}^{2}\right)\left(|\vec{l}|^{2}+m_{\pi}^{2}\right)}+\ldots\right\} \\
= & 3-5 x \tan ^{-1}\left(\frac{1}{x}\right)+\frac{x^{2}}{1+x^{2}}+\frac{x^{2}}{\sqrt{1+2 x^{2}}} \tan ^{-1}\left(\frac{\sqrt{1+2 x^{2}}}{x^{2}}\right),
\end{aligned}
$$

with $A=(4 \pi)^{5} / 2 p_{F}^{3}(n)$, and $x \equiv m_{\pi} / 2 p_{F}(n)$. The level of nucleon degeneracy is characterised by the $|\vec{k} \cdot \vec{l}|^{2}$ term. In the case of strong degeneracy, $|\vec{k} \cdot \vec{l}|^{2}=0$. Note also that in the degenerate limit, the pion mass terms $m_{\pi}^{2}$ in the braces cannot be neglected. Finally performing the integral over the nucleon energies yields

$$
\int \prod_{i=1}^{4} d E_{i} f_{1} f_{2}\left(1-f_{3}\right)\left(1-f_{4}\right) \delta\left(E_{1}+E_{2}-E_{3}-E_{4}-\omega\right)=T^{3} J_{\alpha \alpha}(y),
$$

with $y \equiv \omega / T$, and

$$
J_{\alpha \alpha}(y)=-\frac{1}{6}\left(y^{3}+4 \pi^{2} y\right)\left(1-e^{y}\right)^{-1}
$$




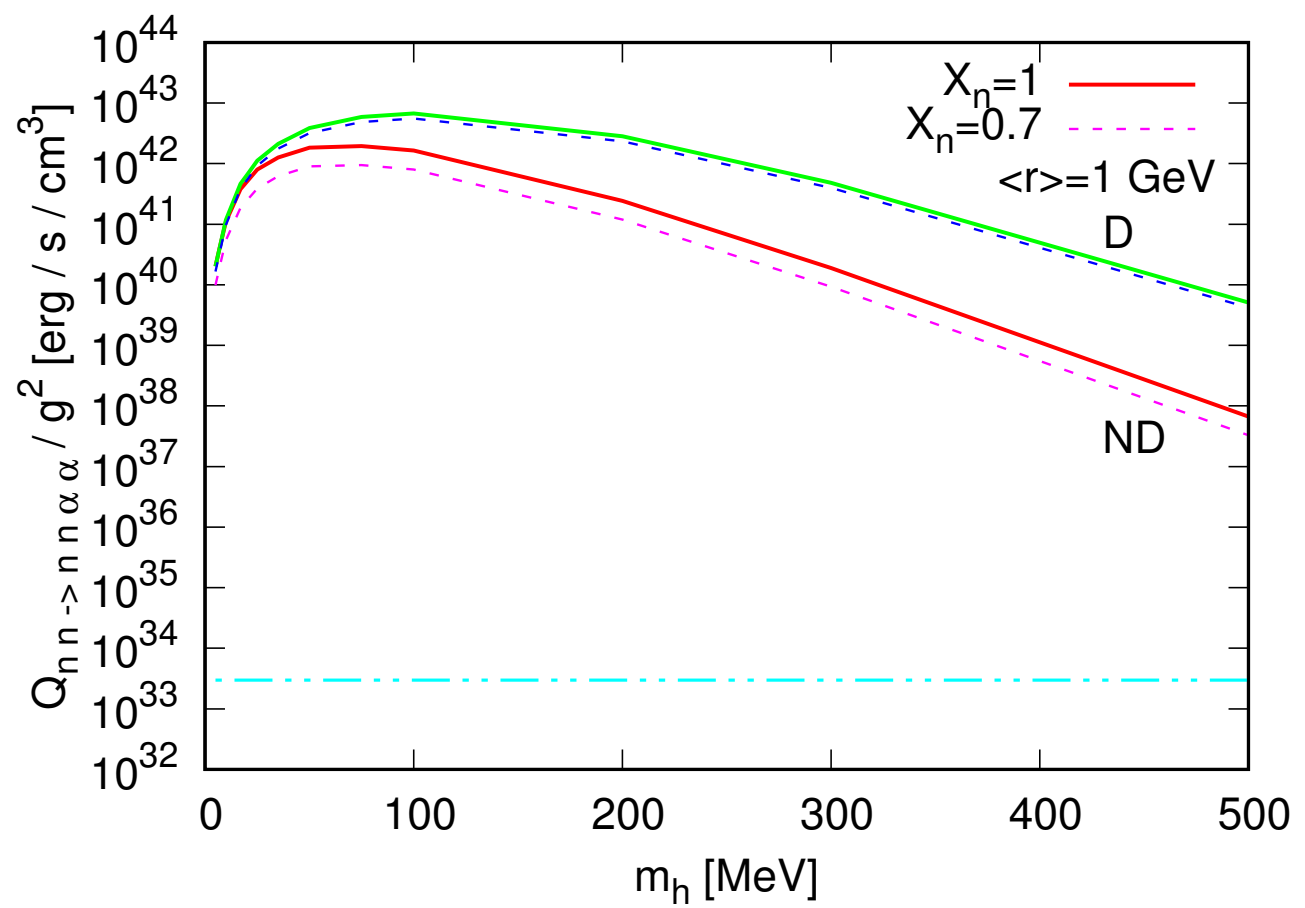

Figure 1. Energy loss rate due to Goldstone boson emission from nuclear bremsstrahlung processes $n n \rightarrow n n \alpha \alpha$ divided by the Higgs portal coupling $g^{2}$, for various light Higgs boson mass $m_{h}$. The rates are calculated in the non-degenerate (ND) and degenerate (D) limits, for proto-neutron star core temperature $T=30 \mathrm{MeV}$, neutron fraction $X_{n}=1$ (solid) and 0.7 (dashed), respectively. For all $m_{h}$ values we assume the radial field vacuum expectation value is $\langle r\rangle=1 \mathrm{GeV}$. Also shown is Raffelt's analytical criterion on the energy loss rate per unit volume $Q_{X}$ in eq. (5.1) (dash-double-dotted).

The energy loss rate in the degenerate limit is then

$$
Q_{N N \rightarrow N N \alpha \alpha}^{\mathrm{OPE}(\mathrm{D})}=\frac{\mathcal{S}}{(2 \pi)^{9}} 4\left\langle F_{N N}\right\rangle I_{\alpha \alpha}\left(\frac{f_{N} g m_{N}}{m_{H}^{2}}\right)^{2}\left(\frac{2 m_{N} f_{\pi}}{m_{\pi}}\right)^{4} p_{F}(n) \frac{T^{8}}{m_{N}^{2}},
$$

with the function given by

$$
I_{\alpha \alpha}\left(m_{h},\langle r\rangle\right) \equiv \int_{0}^{\infty} d y y^{4} I_{1}\left(y, m_{h},\langle r\rangle\right) J_{\alpha \alpha}(y) .
$$

We evaluate $I_{\alpha \alpha}$ numerically using the VEGAS subroutine both directly and using the limit of the Poisson kernel, eq. (3.13). Here we also checked that Goldstone boson production can be well described by the production of a real light Higgs boson and its subsequent decay. We compare the results in these two limits at the nuclear saturation density $\rho=3 \cdot 10^{14} \mathrm{~g} / \mathrm{cm}^{3}$. In figure 1 the comparison is made at the PNS core temperature $T=30 \mathrm{MeV}$ and neutron fraction $X_{n}=1$ and 0.7. Energy loss rate calculated in the two limits have different dependence on $X_{n}: Q_{N N \rightarrow N N \alpha \alpha}^{(\mathrm{ND})} \propto X_{n}^{2}$, and $Q_{N N \rightarrow N N \alpha \alpha}^{(\mathrm{D})} \propto X_{n}^{1 / 3}$. In figure 2 the comparison is made at two different PNS core temperature $T=30 \mathrm{MeV}$ and $20 \mathrm{MeV}$.

It was pointed out that in the case of a mixture of neutrons and protons, in the degenerate limit the energy loss rate for $n p \rightarrow n p \alpha \alpha$ dominates that for $n n \rightarrow n n \alpha \alpha$ and 


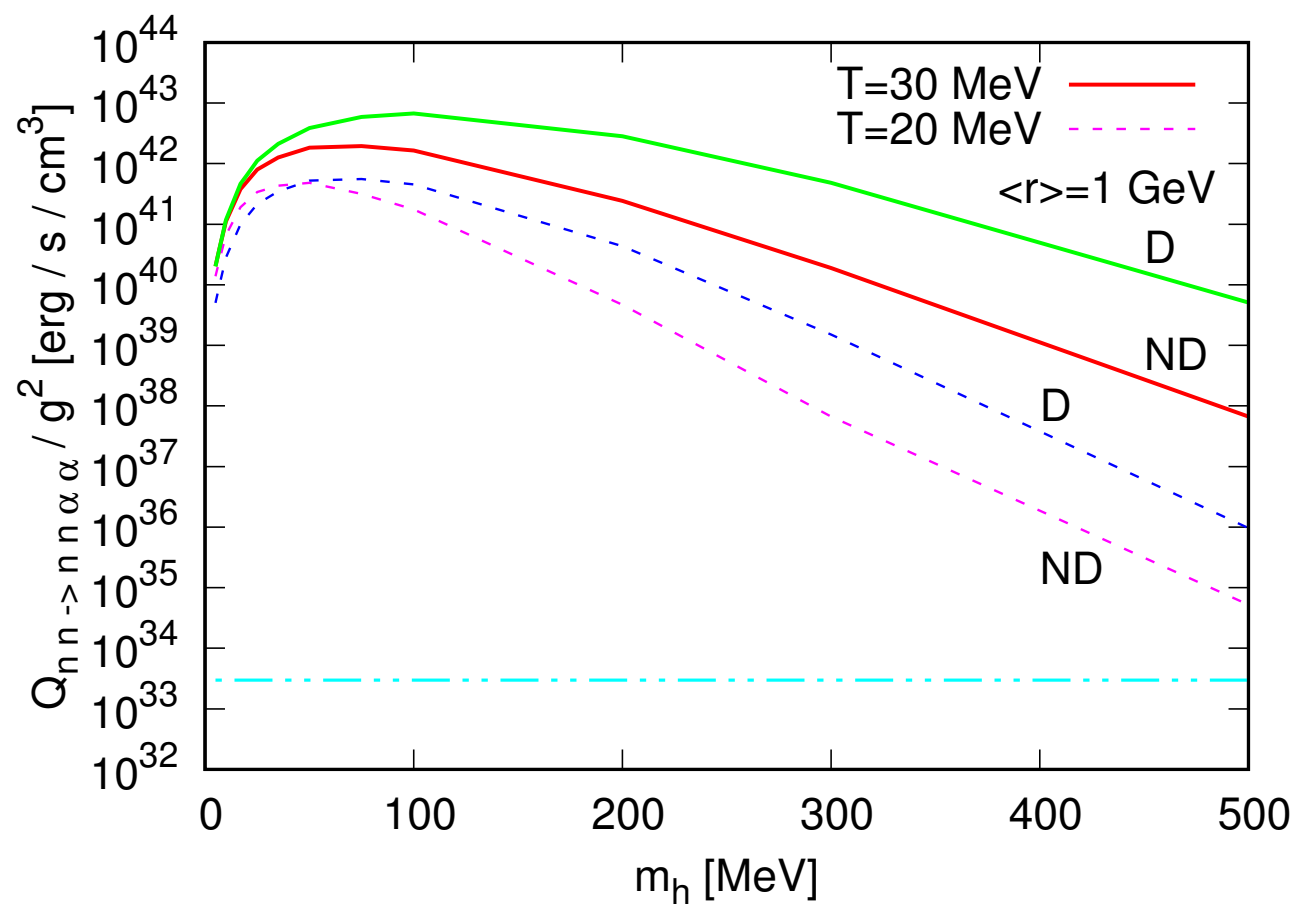

Figure 2. Energy loss rate due to Goldstone boson emission from nuclear bremsstrahlung processes $n n \rightarrow n n \alpha \alpha$ divided by the Higgs portal coupling $g^{2}$, for various light Higgs boson mass $m_{h}$. The rates are calculated in the non-degenerate (ND) and degenerate (D) limits, for proto-neutron star core temperature $T=30 \mathrm{MeV}$ (solid) and $20 \mathrm{MeV}$ (dashed), respectively, and neutron fraction $X_{n}=1$. For all $m_{h}$ values we assume the radial field vacuum expectation value is $\langle r\rangle=1 \mathrm{GeV}$. Also shown is Raffelt's analytical criterion on the energy loss rate per unit volume $Q_{X}$ in eq. (5.1) (dash-double-dotted).

$p p \rightarrow p p \alpha \alpha$, for all lepton fraction $Y_{p}$ values. In ref. [12] the axion emission rate was evaluated numerically for arbitrary neutron degeneracies. It was found therein that the non-degenerate, analytical rate is a very good approximation. More recently, neutrino processes in post-collapse supernova core was studied in the partially-degenerate regime in ref. [65]. In this work we consider $n n$ interactions with $X_{n}=1$ in the non-degenerate limit.

\subsection{Energy loss rate using phase shifts data}

One can also use the experimentally measured cross sections for $N N$ elastic scattering to obtain amplitude estimates for the nuclear bremsstrahlung processes. Many independent observables are available from the nucleon-nucleon elastic scattering data collected by the EDDA Experiment at the Cooler Synchrotron (COSY) in Jülich [66, 67], experiments at the SATURNE II accelerator at Saclay, at the PSI, Ohio University, JINR, TSL in Uppsala, TUNL etc. (see e.g. refs. [68,69].) In $N N$ interactions, the values of the total spin $\vec{S}$ and total angular momentum $\vec{J}=\vec{L}+\vec{S}$ are conserved, but that of the orbital angular momentum $\vec{L}$ may change because of the tensor force. Therefore for $S=1$, partial wave states $\ell_{<}=|J-1|$ and $\ell_{>}=J+1$ can couple to each other. In this case the scattering S-matrix has a $2 \times 2$ matrix structure, parametrised by the mixing angle $\epsilon_{J}$. The 
diagonal elements are given by $e^{2 i \delta_{\ell_{<}}} \cos 2 \epsilon_{J}$ and $e^{2 i \delta_{\ell}} \cos 2 \epsilon_{J}$, respectively, while the offdiagonal elements are both $i e^{i\left(\delta_{\ell_{<}}+\delta_{\ell_{>}}\right)} \sin 2 \epsilon_{J}$. Phase shifts $\delta_{\ell S J}$ and mixing angles $\epsilon_{J}$ for a wide range of laboratory kinetic energies $T_{\text {lab }}$ are available at the Nijmegen NN-OnLine website [70]. Full data and a number of fits to data are available on the SAID database [71]. In the energy range below $25 \mathrm{MeV}$, there are numerous measurements on the total $n p$ cross section, but not on $p p$ due to the large Coulomb effects. Therefore the uncertainties in the latter are larger.

A nice summary of the general formalism for two-body scattering of spin- $1 / 2$ particles can be found in ref. [72]. The total cross section for $p p$ elastic scattering is simply

$$
\sigma_{N N}=2 \pi \sum_{J}(2 J+1)\left|f_{J}\left(\vec{k}_{\mathrm{cm}}\right)\right|^{2}=\frac{2 \pi}{\left|\vec{k}_{\mathrm{cm}}\right|^{2}} \sum_{J}(2 J+1) \sin ^{2} \delta_{\ell S J}\left(\vec{k}_{\mathrm{cm}}\right),
$$

where $f_{J}$ 's are the partial-wave amplitudes, and $\vec{k}_{\mathrm{cm}}$ is the momentum in the centre-of-mass system. It is related to the laboratory kinetic energy as $\left|\vec{k}_{\mathrm{cm}}\right|^{2}=\frac{1}{2} m_{p} T_{\mathrm{lab}}$, with $m_{p}$ the proton mass.

\subsubsection{Global fits of total elastic cross sections}

In this work we use the SP07 and LE08 global fits for the total proton-proton and neutronproton elastic scattering cross sections $\sigma_{p p}$ and $\sigma_{n p}$ [69, 73], respectively, as shown in figure 3. The errors quoted therein are quite small, ranging from $0.01 \mathrm{mb}$ for low incident energies to $0.8 \mathrm{mb}$ at most for high incident energies. The huge cross section at zero-energy indicates that there is a two-body bound state, or quasi-bound state, as manifested in the negative scattering lengths $a_{p p} \approx-17.1 \mathrm{fm}$ and $a_{n p} \approx-23.74 \mathrm{fm}$ (see e.g. ref. [47].) We also plot the $N N$ elastic scattering cross section calculated using the OPE approximation, where for simplicity we neglect the pion mass $m_{\pi}$ in the braces in the amplitude expression

$$
\begin{aligned}
\sum_{\text {spins }}\left|\mathcal{M}_{N N \rightarrow N N}^{\mathrm{OPE}}\right|^{2}= & 4\left(\frac{2 m_{N} f_{\pi}}{m_{\pi}}\right)^{4}\left\{\frac{|\vec{k}|^{4}}{\left(|\vec{k}|^{2}+m_{\pi}^{2}\right)^{2}}+\frac{|\vec{l}|^{4}}{\left(|\vec{l}|^{2}+m_{\pi}^{2}\right)^{2}}\right. \\
& \left.+\frac{|\vec{k}|^{2}|\vec{l}|^{2}+2(\vec{k} \cdot \vec{l})^{2}-2\left(|\vec{k}|^{2}+|\vec{l}|^{2}\right)(\vec{k} \cdot \vec{l})}{\left(|\vec{k}|^{2}+m_{\pi}^{2}\right)\left(|\vec{l}|^{2}+m_{\pi}^{2}\right)}\right\} .
\end{aligned}
$$

As expected, the OPE approximation is good only for $T_{\text {lab }} \simeq 10-20 \mathrm{MeV}$. For larger laboratory kinetic energies, it overetimates by a factor of 10 (for $T_{\text {lab }} \simeq 100-400 \mathrm{MeV}$ ) to 4 (for $\left.T_{\text {lab }} \simeq 800-1000 \mathrm{MeV}\right)$.

Results in ref. [66] show that for low energy scattering, $d \sigma_{N N} / d \Omega$ has no strong angular dependence. Therefore we simply use $\sum_{\text {spins }}\left|\mathcal{M}_{N N}\right|^{2} \approx 64\left|\mathcal{A}_{N N}\right|^{2} m_{N}^{4}$ to infer $\left|\mathcal{A}_{N N}\right|^{2}$ as a function of the center-of-mass energy $E_{\mathrm{cm}}^{2} \approx 4 m_{N}^{2}+2 m_{N} T_{\text {lab. }}$. With this information, we estimate the amplitude squared for the nuclear bremsstrahlung processes $N N \rightarrow N N \alpha \alpha$

$$
\sum_{\text {spins }}\left|\mathcal{M}_{N N \rightarrow N N \alpha \alpha}^{\exp }\right|^{2} \approx 1024\left|\mathcal{A}_{N N}\right|^{2}\left(\frac{f_{N} g m_{N}}{m_{H}^{2}}\right)^{2} \frac{\left(q_{1} \cdot q_{2}\right)^{2}}{\left(q^{2}-m_{h}^{2}\right)^{2}+m_{h}^{2} \Gamma_{h}^{2}} \frac{\left(-2 q^{2}\right)^{2}}{(2 p \cdot q)^{4}} m_{N}^{6}
$$




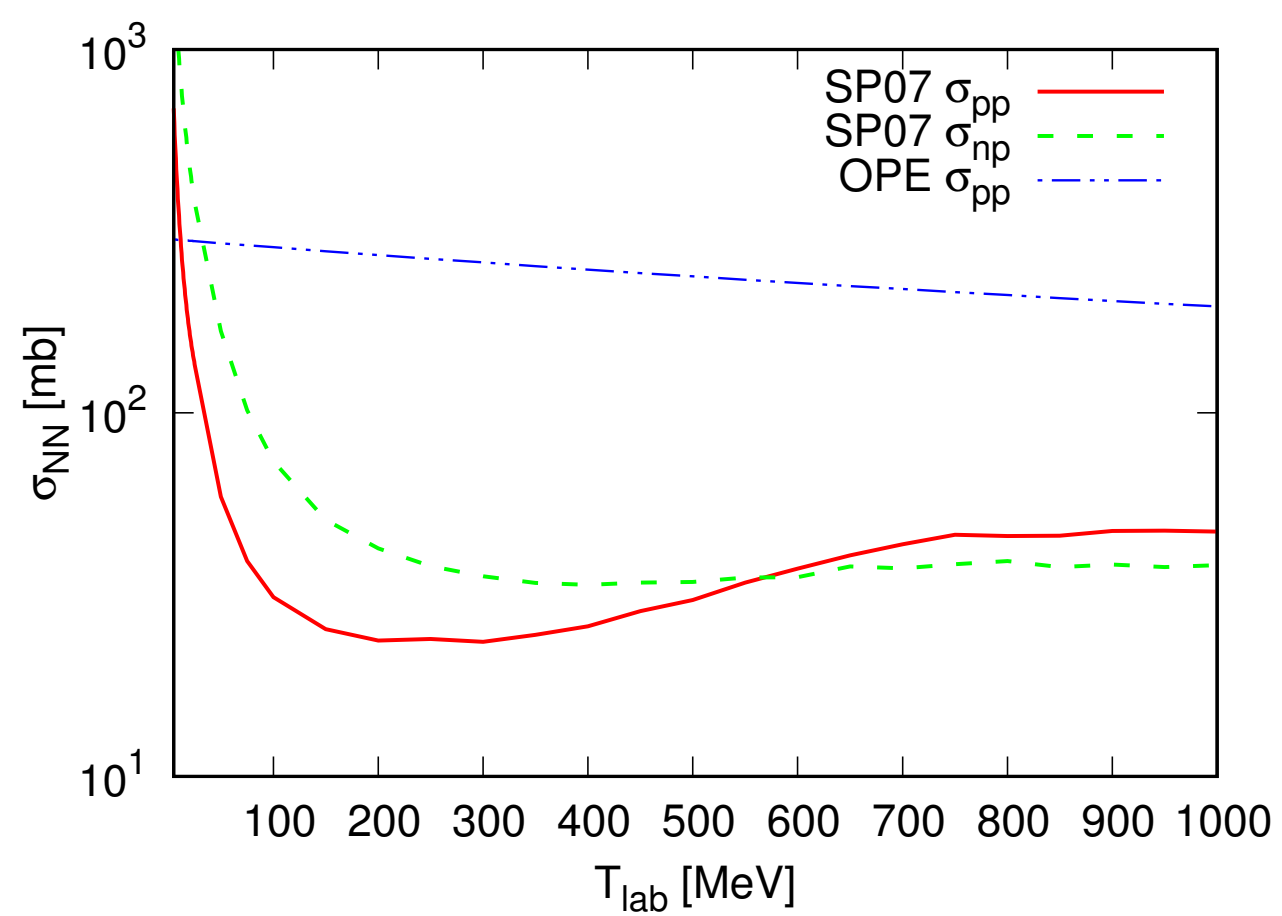

Figure 3. The SP07 global fits for the total $p p$ (solid) and $n p$ (dashed) elastic scattering cross sections as a function of the laboratory kinetic energy $T_{\text {lab }}$, reported in ref. [73]. Also plotted is the total $p p$ elastic cross section obtained using the one-pion exchange (OPE) approach (dash-doubledotted), with the pion mass term $m_{\pi}$ in the braces neglected.

after summing over 64 direct and exchange diagrams. To evaluate the phase space integral in the energy loss rate calculation, we take the non-degenerate limit, and proceed as in the OPE case. The energy loss rate is then

$$
Q_{N N \rightarrow N N \alpha \alpha}^{\exp (\mathrm{ND})}=\frac{32 \mathcal{S}}{(2 \pi)^{6}} I_{0}^{\exp } n_{N}^{2}\left(\frac{f_{N} g m_{N}}{m_{H}^{2}}\right)^{2} \frac{T^{11 / 2}}{m_{N}^{9 / 2}} .
$$

Here we define the integral

$$
\begin{aligned}
I_{0}^{\exp }\left(T, m_{h},\langle r\rangle\right) \equiv \int & d u d v d x d y x^{4} I_{1}\left(x, T, m_{h},\langle r\rangle\right) \sqrt{y} e^{-y} \sqrt{u v} e^{-u} \delta(u-v-x) \\
& \times m_{N}^{4}\left|\mathcal{A}_{N N}\right|^{2}(u, y),
\end{aligned}
$$

with $y \equiv|\vec{P}|^{2} / m_{N} T$. The result obtained by using the SP07 global fit to the $\sigma_{p p}$ data is plotted in figure 4 and compared to the OPE result. The overestimation by OPE happens to be milder for $N N \rightarrow N N \alpha \alpha$ than in $N N \rightarrow N N$, because of the different kinematics of the exchanged pion in the nuclear bremsstrahlung processes from that in the elastic scattering.

For neutrino emission from the $n n \rightarrow n n \nu \bar{\nu}$ processes, ref. [59] used on-shell $N N$ amplitudes measured by experiments and found that the OPE approximation overestimated the energy loss rate by about a factor of four. In ref. [54] it was found that the nextto-next-to-next-to-leading order $\left(\mathrm{N}^{3} \mathrm{LO}\right)$ chiral effective field theory calculations differ by about a factor 2-3 from leading order (LO) results, and the result obtained by using the 


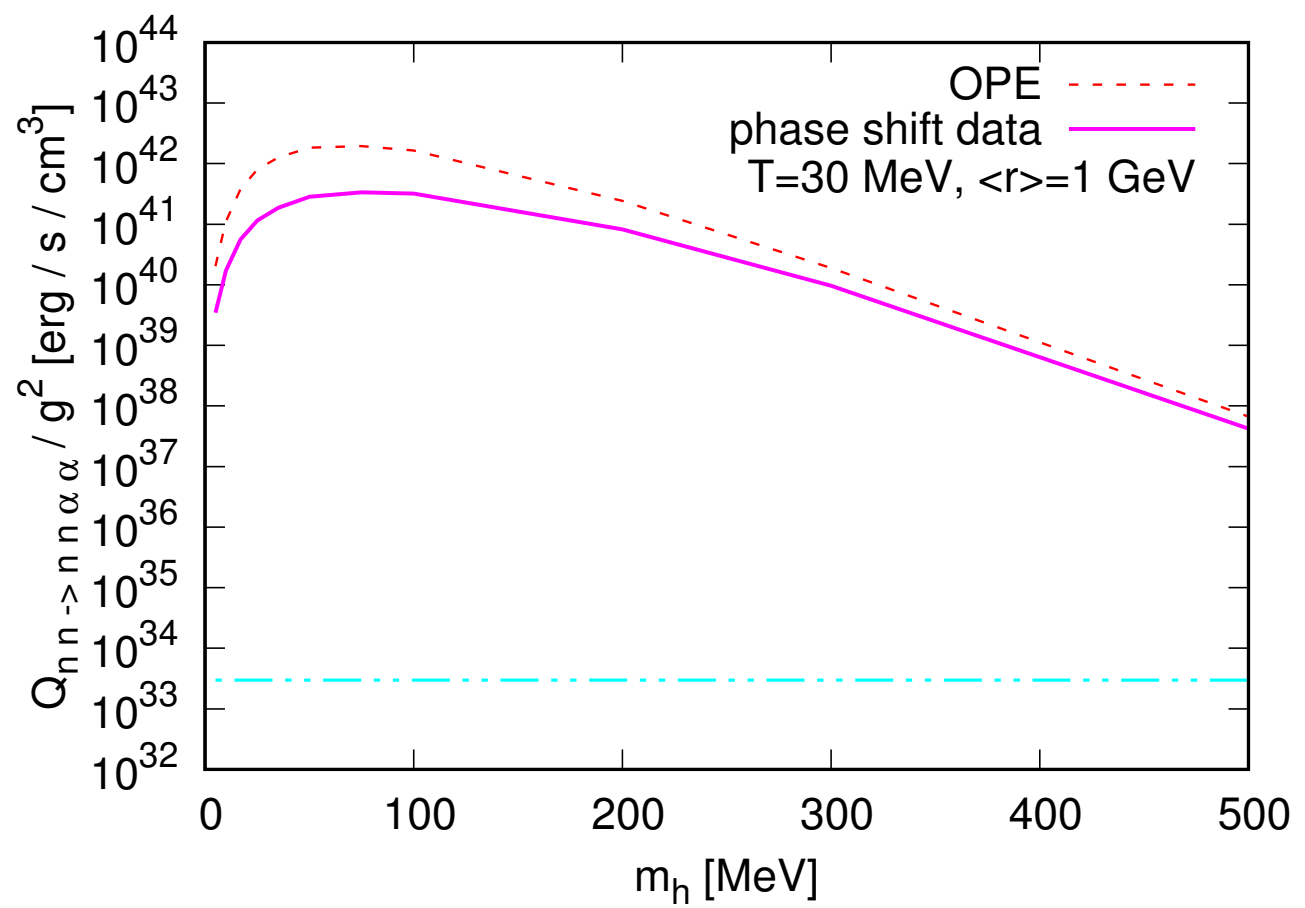

Figure 4. Energy loss rate due to Goldstone boson emission from nuclear bremsstrahlung processes $n n \rightarrow n n \alpha \alpha$ divided by the Higgs portal coupling $g^{2}$, for various light Higgs boson mass $m_{h}$. The rates are calculated using the one-pion exchange (OPE) approximation (dashed) and the SP07 global fits for the total $p p$ elastic cross section (solid), and assume charge symmetry, i.e. $\sigma_{n n}=\sigma_{p p}$. Here we take the non-degenerate (ND) limit only, and set proto-neutron star core temperature $T=30 \mathrm{MeV}$ and neutron fraction $X_{n}=1$. For all $m_{h}$ values we assume the radial field vacuum expectation value is $\langle r\rangle=1 \mathrm{GeV}$. Also shown is Raffelt's analytical criterion on the energy loss rate per unit volume $Q_{X}$ in eq. (5.1) (dash-double-dotted).

experimental phase shifts data is very similar to the $\mathrm{N}^{3} \mathrm{LO}$ ones. Since the central contact terms in the chiral effective field theory do not contribute in the nuclear bremsstrahlung processes, the leading-order term is solely the one-pion exchange potential. For axions, the OPE approximation is also found to oversimplify the nuclear dynamics and overestimate the emission rate by a factor of four [59].

\subsubsection{Chiral effective field theory predictions}

Charge independence breaking (CIB) of the strong $N N$ interactions refers to the difference between the isospin $I=1$ states: the proton-proton $\left(I_{z}=+1\right)$, the neutron-proton $\left(I_{z}=0\right)$, and the neutron-neutron $\left(I_{z}=-1\right)$ interactions, after electromagnetic effects are removed. Charge symmetry breaking (CSB) concerns the difference between the $p p$ and $n n$ interactions only. CIB is clearly seen in figure 3, while a small amount of CSB is observed in the measured scattering lengths $a_{n n}$ and $a_{p p}$, as well as the effective ranges $r_{n n}$ and $r_{p p}$. A detailed discussion on charge dependence of nuclear interactions can be found in ref. [51] (see also ref. [74].) Very recently, ref. [47] provides $p p, n n$ and $n p$ phase shifts predicted by the chiral effective field theory to the $\mathrm{N}^{4} \mathrm{LO}$. In all partial waves, the predicted $n p$ phase shifts and mixing angles at this order are shown to agree excellently with the Nijmegen 


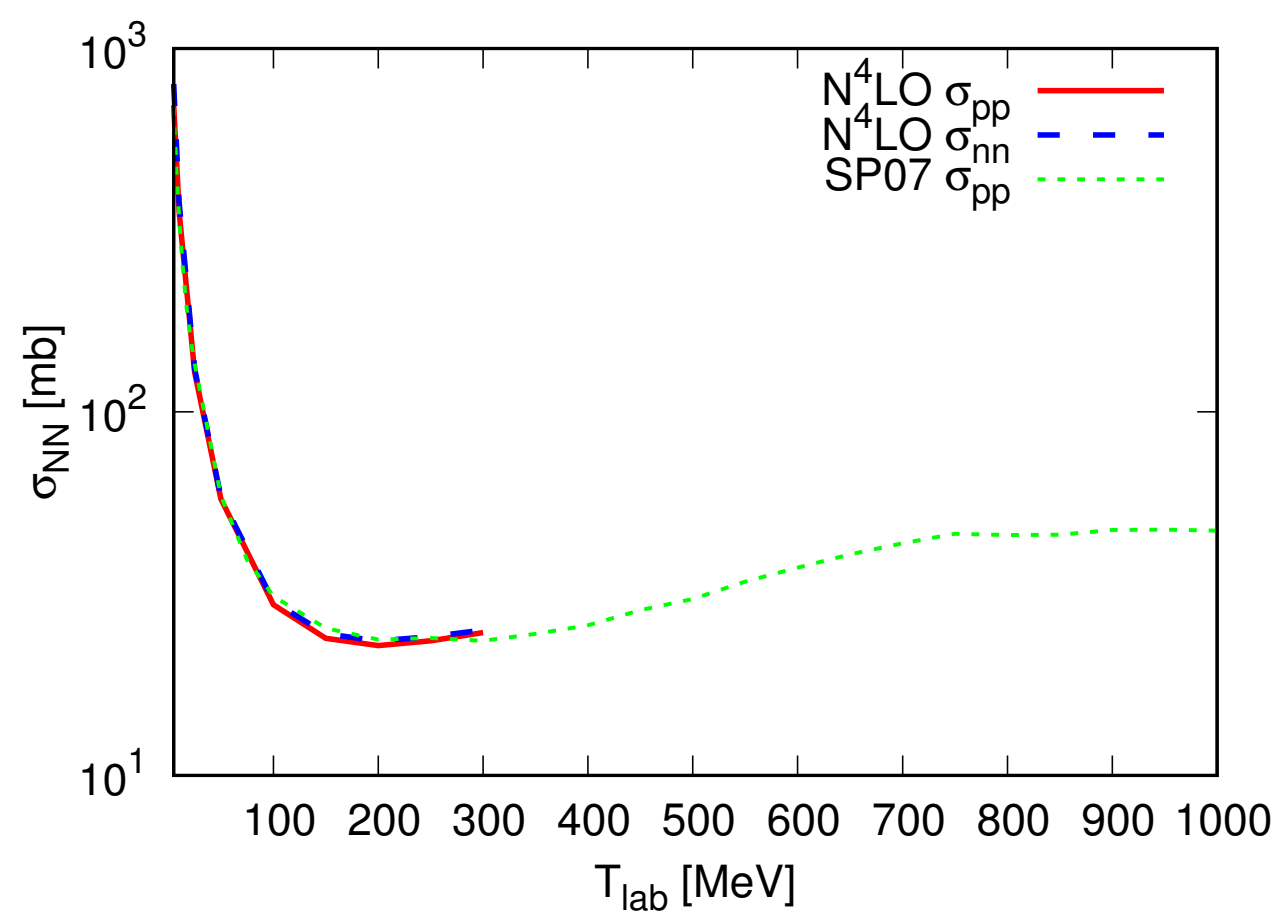

Figure 5. Total $p p$ (solid) and $n n$ (dashed) elastic scattering cross sections as a function of the lab kinetic energy $T_{\text {lab }}$, from the $\mathrm{N}^{4} \mathrm{LO}$ chiral effective field theory results for the phase shifts presented in ref. [47]. Also plotted is the SP07 global fits for the total $p p$ elastic cross section (dotted) reported in ref. [73].

multi-energy [75] and the SP07 single-energy analysis [69]. Charge-dependence due to pionmass splitting is taken into account in the one-pion exchange terms only, while nucleonmass splitting is always included. The total $p p$ and $n n$ elastic cross sections calculated with eq. (3.21) using the $\mathrm{N}^{4} \mathrm{LO}$ chiral effective field theory phase shifts from ref. [47] are shown in figure 5 . The $p p$ results agree very well with the SP07 global fit results. For $T_{\text {lab }} \lesssim 10 \mathrm{MeV}$, Coulomb force in $p p$ collisions is significant. At larger laboratory kinetic energies, chiral effective field theory calculations predict that the effects of charge symmetry breaking is $\lesssim 3 \%$ only. In this work we therefore use the experimental data and set $\sigma_{n n}=\sigma_{p p}$.

Low-energy theorems [76-79] state that the first two terms in the series expansion of the bremsstrahlung amplitude in powers of the energy loss may be exactly calculated by using the corresponding elastic, i.e. non-radiative, amplitude. In ref. [23] it was argued that the model-independent approach of relating the nuclear bremsstrahlung amplitudes to the on-shell $N N$ scattering amplitudes measured by experiments is not applicable to scalar particles such as the saxion. The reason is that the contributions to the leading order terms $\left(\propto \omega^{-1}\right)$ from the emission of a scalar particle from external nucleon legs cancel each other, which does not happen for axion and neutrino pairs [59], or KK-gravitons [14]. The next-to-leading order term $\left(\propto \omega^{0}\right)$ includes the emission diagrams of the scalar particle from external legs as well as from internal lines, where the latter is not calculable due to the unknown interaction vertices, and may be dominant. 
In Weinberg's Higgs portal model, we also found the cancellation of the leading order terms between the diagrams for the Goldstone boson pairs being emitted from the external nucleon legs. The effective Higgs-pion coupling is $\propto\left(q^{2}+\frac{11}{2} m_{\pi}^{2}\right) /\langle\varphi\rangle$, so the emission from internal lines is of order $\mathcal{O}\left(\omega^{0}\right)$ as well in the low-energy limit (cf. eq. (3.3)). However, in Weinberg's Higgs portal model Goldstone boson production in the PNS core is dominated by the emission of a real light Higgs boson in nuclear bremsstrahlung processes and its subsequent decay. Therefore for small light Higgs boson mass $m_{h}$ the low-energy theorems should still be applicable. This remains to be verified by using the chiral effective field theory to calculate the emission of the light Higgs boson $h$ from the external nucleon legs as well as from the internal lines.

\section{Goldstone boson propagation in proto-neutron star core}

In the weakly-interacting regime, the Goldstone boson mean free path is set by the elastic scattering rate $R_{\alpha N \rightarrow \alpha N}$. In the strongly-interacting regime, the absorption rate $R_{N N \alpha \alpha \rightarrow N N}$ may be comparable. The mean free path in the former case is $l_{\mathrm{mfp}}=\left(n_{N} \sigma_{\alpha N \rightarrow \alpha N}\right)^{-1}$, while in the latter case, the mean free path against absorption is $l_{\mathrm{mfp}}^{\text {absorb. }}=\left(n_{N}^{2} \sigma_{\alpha \alpha N N \rightarrow N N}\right)^{-1}$. For axions, ref. [10] has considered the free-streaming regime, while ref. [80] the trapping regime.

The amplitude squared for the elastic process $\alpha\left(q_{1}\right) N\left(p_{1}\right) \rightarrow \alpha\left(q_{2}\right) N\left(p_{2}\right)$ is

$$
\sum_{\text {spins }}\left|\mathcal{M}_{\alpha N \rightarrow \alpha N}\right|^{2}=\frac{4 f_{N}^{2} g^{2} m_{N}^{2}}{m_{H}^{4}} \frac{\left(q_{1} \cdot q_{2}\right)^{2}\left[\left(p_{1} \cdot p_{2}\right)+m_{N}^{2}\right]}{\left(t-m_{h}^{2}\right)^{2}},
$$

where $t=\left(q_{2}-q_{1}\right)^{2}=\left(p_{1}-p_{2}\right)^{2}$. We follow ref. [81] to calculate the reaction rate

$$
\begin{aligned}
R_{\alpha N \rightarrow \alpha N}= & n_{N} \sigma_{\alpha N \rightarrow \alpha N} v_{\mathrm{M}}=\int \frac{2 d^{3} \vec{p}_{1}}{(2 \pi)^{3}} f\left(\vec{p}_{1}\right) \frac{1}{2 \omega_{1} 2 E_{1}} \int \frac{d^{3} \vec{q}_{2}}{(2 \pi)^{3} 2 \omega_{2}} \\
& \times \int \frac{d^{3} \vec{p}_{2}}{(2 \pi)^{3} 2 E_{2}}\left[1-f\left(\vec{p}_{2}\right)\right] \frac{1}{2} \sum_{\text {spins }}\left|\mathcal{M}_{\alpha N \rightarrow \alpha N}\right|^{2}(2 \pi)^{4} \delta^{4}\left(p_{1}+q_{1}-p_{2}-q_{2}\right),
\end{aligned}
$$

where $v_{\mathrm{M}}$ is the Møller velocity. Using the polar angle $\cos \theta \equiv \vec{p}_{1} \cdot \vec{q}_{1} /\left|\vec{p}_{1}\right|\left|\vec{q}_{1}\right|$ and the azimuthal angel $\phi^{\prime}$ which is measured from the $\left(\vec{p}_{1}, \vec{q}_{1}\right)$-plane, the 9-dimensional integral can be simplified to

$$
\begin{aligned}
R_{\alpha N \rightarrow \alpha N}= & \frac{1}{(2 \pi)^{3}} \frac{m_{N}^{4}}{4 \omega_{1}} \frac{f_{N}^{2} g^{2} m_{N}^{2}}{m_{H}^{4}} \int_{1}^{\infty} d \epsilon_{1} f\left(\epsilon_{1}\right) \sqrt{\epsilon_{1}^{2}-1} \int_{-1}^{+1} \frac{d \cos \theta}{\lambda\left(\epsilon_{1}, u_{1}, \cos \theta\right)} \\
& \times \int_{\epsilon_{2}^{\min }}^{\epsilon_{2}^{\max }} d \epsilon_{2}\left[1-f\left(\epsilon_{2}\right)\right] \int_{0}^{2 \pi} \frac{d \phi^{\prime}}{2 \pi} F_{3}
\end{aligned}
$$

with the dimensionless variables $\epsilon_{1} \equiv E_{1} / m_{N}, \epsilon_{2} \equiv E_{2} / m_{N}$, and $u_{1} \equiv \omega_{1} / m_{N}$. The functions in the above equation are defined as

$$
\lambda\left(\epsilon_{1}, u_{1}, \cos \theta\right) \equiv \frac{\left|\vec{p}_{1}+\vec{q}_{1}\right|}{m_{N}}=\sqrt{\epsilon_{1}^{2}-1+u_{1}^{2}+2 u_{1}\left(\epsilon_{1}^{2}-1\right)^{1 / 2} \cos \theta},
$$


and

$$
F_{3} \equiv \frac{\left[q_{1} \cdot\left(p_{1}+q_{1}-p_{2}\right)\right]^{3}+2 m_{N}^{2}\left[q_{1} \cdot\left(p_{1}+q_{1}-p_{2}\right)\right]^{2}}{\left[2 q_{1} \cdot\left(p_{1}+q_{1}-p_{2}\right)+m_{h}^{2}\right]^{2} m_{N}^{2}},
$$

respectively, and the limits for the $d \epsilon_{2}$ integration are determined to be

$$
\epsilon_{2}^{\max , \min }=\frac{1}{2}\left[\epsilon_{1}+u_{1} \pm \lambda\left(\epsilon_{1}, u_{1}, \cos \theta\right)+\frac{1}{\epsilon_{1}+u_{1} \pm \lambda\left(\epsilon_{1}, u_{1}, \cos \theta\right)}\right] .
$$

To evaluate $q_{1} \cdot p_{2}$, we need to know the angle

$$
\cos \theta_{q_{1} p_{2}} \equiv \cos \theta^{\prime} \cos \Delta_{2}-\sin \theta^{\prime} \sin \Delta_{2} \cos \phi^{\prime}
$$

where

$$
\cos \Delta_{1}=\frac{\sqrt{\epsilon_{1}^{2}-1}+u_{1} \cos \theta}{\lambda}, \quad \cos \Delta_{2}=\frac{u_{1}+\sqrt{\epsilon_{1}^{2}-1} \cos \theta}{\lambda},
$$

with $\Delta_{1}+\Delta_{2}=\theta$, and

$$
\cos \theta^{\prime}=\frac{E_{2}\left(E_{1}+\omega_{1}\right)-p_{1} \cdot q_{1}-m_{N}^{2}}{\left|\overrightarrow{p_{2}}\right|\left|\overrightarrow{p_{1}}+\overrightarrow{q_{1}}\right|}
$$

We evaluate eq. (4.3) numerically using the VEGAS subroutine. For low incident Goldstone boson energies $\omega_{1} \ll m_{N}$, the nuclear recoil effects can be neglected, and so the interaction rate can also be easily estimated by

$$
R_{\alpha N \rightarrow \alpha N} \approx n_{N} \frac{\omega_{1}^{4}}{16 \pi} \frac{f_{N}^{2} g^{2}}{m_{H}^{4}} \int_{-1}^{+1} d \cos \theta \frac{\omega_{1}^{2}(1-\cos \theta)^{3}+2 m_{N}^{2}(1-\cos \theta)^{2}}{\left[2 \omega_{1}^{2}(1-\cos \theta)+m_{h}^{2}\right]^{2}} .
$$

We found that the results from this method agree with those from the full calculation within $20 \%$ for $\omega_{1} \lesssim 100 \mathrm{MeV}$. In figure 6 we plot the Goldstone boson mean free path $l_{\text {mfp }}$ times the Higgs portal coupling $g^{2}$ versus the light Higgs boson mass $m_{h}$, for various incident Goldstone boson energies $\omega_{1}$.

Goldstone boson pairs are emitted with an average energy of

$$
\frac{\bar{\omega}}{T}=\frac{1}{T} \frac{Q_{N N \rightarrow N N \alpha \alpha}}{n_{N}^{2}\left\langle\sigma_{N N \rightarrow N N \alpha \alpha} v_{\mathrm{M}}\right\rangle} .
$$

In figure 7 we choose to plot the ratio of the Goldstone boson average emission energy to the light Higgs boson mass $m_{h}$. The curve indicates again that for $m_{h} \lesssim 500 \mathrm{MeV}$, Goldstone boson emission is dominated by the production of a real light Higgs boson $h$.

We divide the free-streaming and the trapping regime by $l_{\mathrm{mfp}} \gg R_{\mathrm{PNS}}$ and $l_{\mathrm{mfp}} \ll R_{\mathrm{PNS}}$, respectively. The neutron star radius is about $10 \mathrm{~km}[82,83]$, depending on the equation of state (see refs. [84, 85] for recent reviews.) But the proto-neutron star radius is about $10-20 \mathrm{~km}$ at post-bounce times $\lesssim 3 \mathrm{~s}$, slightly larger than that of neutron stars, as shown in the simulations of e.g. ref. [2]. Therefore, if the Higgs portal coupling saturates the collider bound $g \leq 0.011$, the Goldstone bosons would be trapped in the PNS core. In this case they still contribute to the cooling of the PNS core, and one needs to estimate the opacity of the medium to the Goldstone bosons as in ref. [80] for axions. The amplitudes 


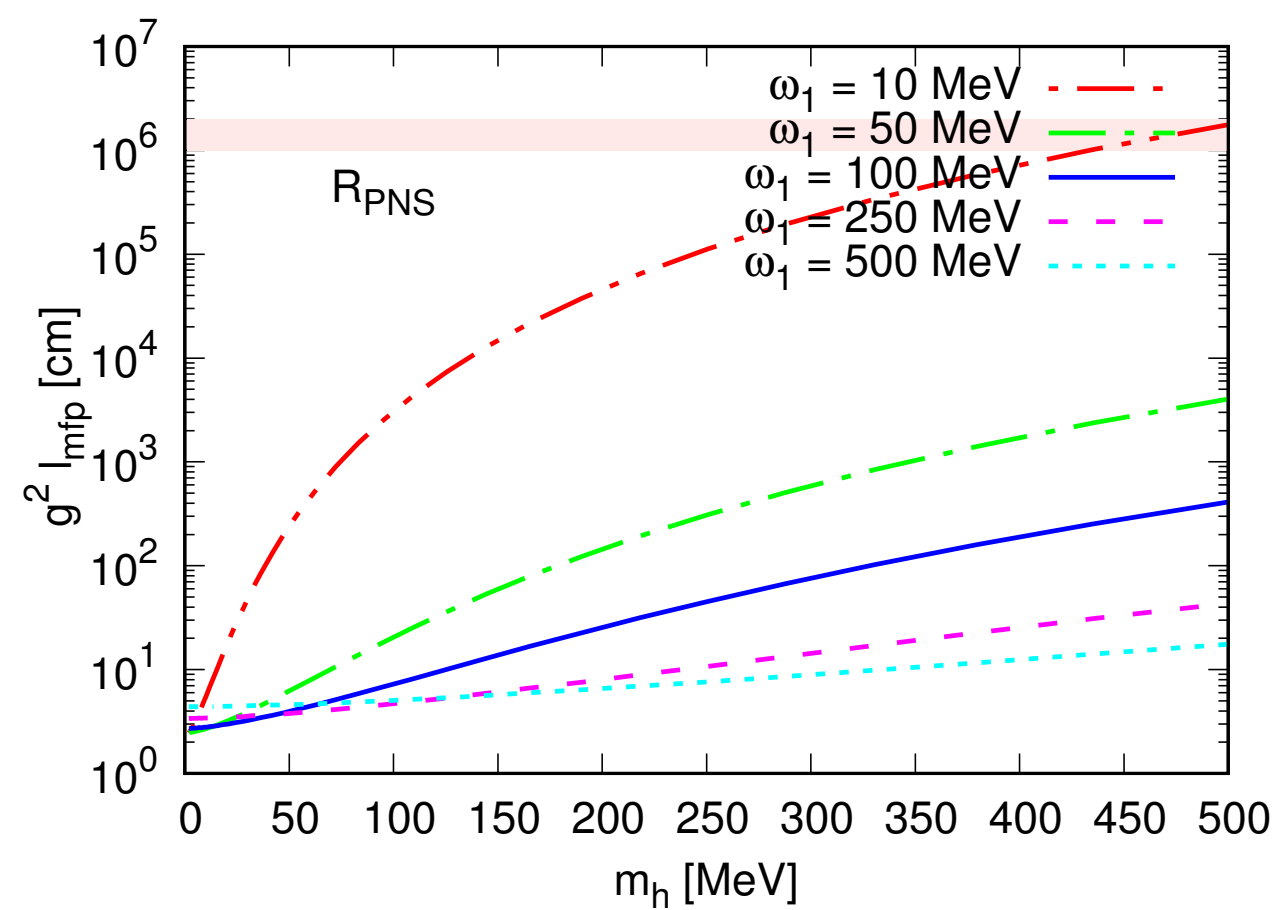

Figure 6. Goldstone boson mean free path $l_{\mathrm{mfp}}$ times the Higgs portal coupling $g^{2}$ in the protoneutron star core versus the light Higgs boson mass $m_{h}$. Here we show the dependence on the incident Goldstone boson energy for the values $\omega_{1}=10$ (dash double-dotted), 50 (dash-dotted), 100 (solid), 250 (dashed), and $500 \mathrm{MeV}$ (dotted), respectively. Also shown is the proto-neutron star radius $R_{\mathrm{PNS}} \approx 10-20 \mathrm{~km}$ (shaded region).

for the Goldstone boson pair absorption rate, $\sum_{\text {spins }}\left|\mathcal{M}_{N N \alpha \alpha \rightarrow N N}\right|^{2}$, are the same as for the nuclear bremsstrahlung energy loss rate. For simplicity, in this work we consider only the free-streaming regime by demanding

$$
g \lesssim \sqrt{\frac{g^{2} l_{\mathrm{mfp}}(\bar{\omega})}{R_{\mathrm{PNS}}}} \equiv g_{\mathrm{fs}},
$$

for each light Higgs boson mass $m_{h}$. We plot the Goldstone boson free-streaming criterion $g_{\mathrm{fs}}$ in figure 8 , assuming $R_{\mathrm{PNS}}=20 \mathrm{~km}$ for the proto-neutron star radius. For $m_{h} \lesssim 50 \mathrm{MeV}$, it is beyond the projected sensitivity of future collider experiments for SM Higgs invisible decay (cf. eq. (2.9)).

\section{SN 1987A constraints on Weinberg's Higgs portal model}

Ideally one should do numerical simulations as in refs. $[15,24,25]$ to study the effects of the additional cooling agent on the neutrino burst signal. Here we simply invoke Raffelt's analytical criterion $[26,27]$ on the energy loss rate per unit mass due to the emission of an exotic species $X$

$$
\epsilon_{X} \equiv \frac{Q_{X}}{\rho} \lesssim 10^{19} \mathrm{erg} \cdot \mathrm{g}^{-1} \cdot \mathrm{s}^{-1}
$$




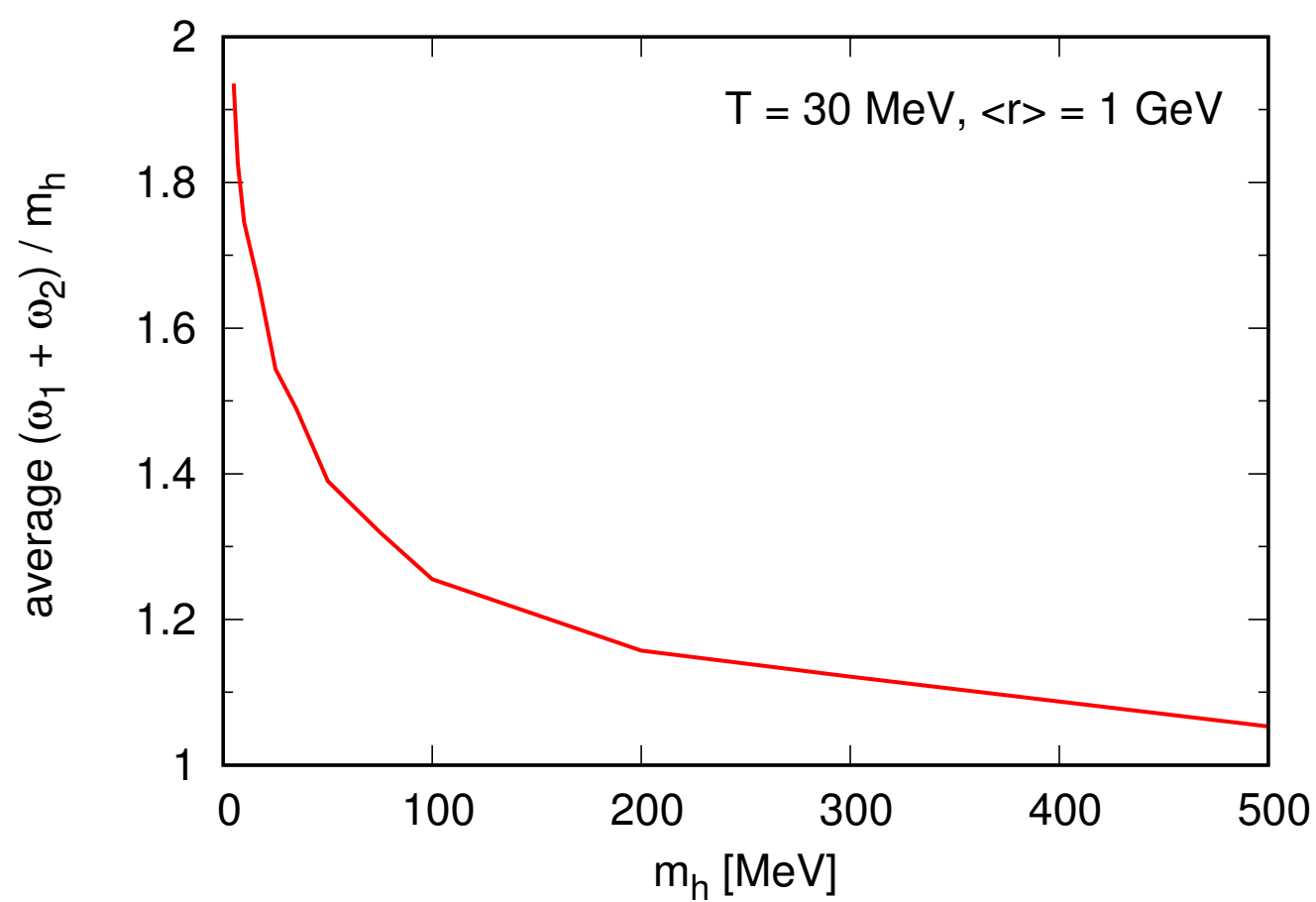

Figure 7. Goldstone boson average emission energy $\bar{\omega}=\overline{\omega_{1}+\omega_{2}}$ in dependence of the light Higgs boson mass $m_{h}$. Both the energy loss rate $Q_{n n \rightarrow n n \alpha \alpha}$ and the thermally averaged cross section $\left\langle\sigma_{n n \rightarrow n n \alpha \alpha}\right\rangle v_{\mathrm{M}}$ are calculated in the non-degenerate (ND) limit, for proto-neutron star core temperature $T=30 \mathrm{MeV}$, neutron fraction $X_{n}=1$, and the radial field vacuum expectation value $\langle r\rangle=1 \mathrm{GeV}$.

as shown in figure 1, figure 2, and figure 4. It is to be applied at typical PNS core conditions, i.e. at a temperature $T=30 \mathrm{MeV}$, and baryon mass density $\rho=3 \cdot 10^{14} \mathrm{~g} / \mathrm{cm}^{3}$. The SN 1987A constraint on Weinberg's Higgs portal model is obtained by finding the model parameters $g$ and $\langle r\rangle$ for each light Higgs boson mass $m_{h}$ such that the energy loss rate due to Goldstone boson emission $Q_{N N \rightarrow N N \alpha \alpha}<Q_{X}$. In the resonance region of producing a real light Higgs boson $h$, where the approximation with Poisson kernel limit is applicable, we have seen that $Q_{N N \rightarrow N N \alpha \alpha} \propto g\langle r\rangle$. Therefore we scale the estimates for this quantity calculated using the one-pion exchange (OPE) approach and the SP07 global fits for the elastic $p p$ cross section to be below $Q_{X}$, where the non-degenerate (ND) limit is taken and charge symmetry, i.e. $\sigma_{n n}=\sigma_{p p}$ (cf. figure 4) is assumed. Our main results are presented in figure 9 . In these SN 1987A constraints, the collider bound and the freestreaming criterion on $g$ (eq. (2.9) and eq. (4.12), respectively), as well as the perturbativity condition on $\langle r\rangle$ (eq. (2.10)) are all satisfied. We find that using OPE and the SP07 global fits results only in a factor of 2.6 difference for $10 \mathrm{MeV} \lesssim m_{h} \lesssim 50 \mathrm{MeV}$, and a factor of 1.4 for $m_{h}>300 \mathrm{MeV}$. Uncertainty from the effective Higgs-nucleon coupling $f_{N}$ is around 10\%. Quantifying and discussing many-body and medium effects, or the impact of nucleon effective masses [86] in nuclear interactions are beyond the scope of this work.

Nevertheless, figure 9 makes clear that with nuclear uncertainties taken into account, the SN 1987A constraints still surpass those set by laboratory experiments [33], or by energy 


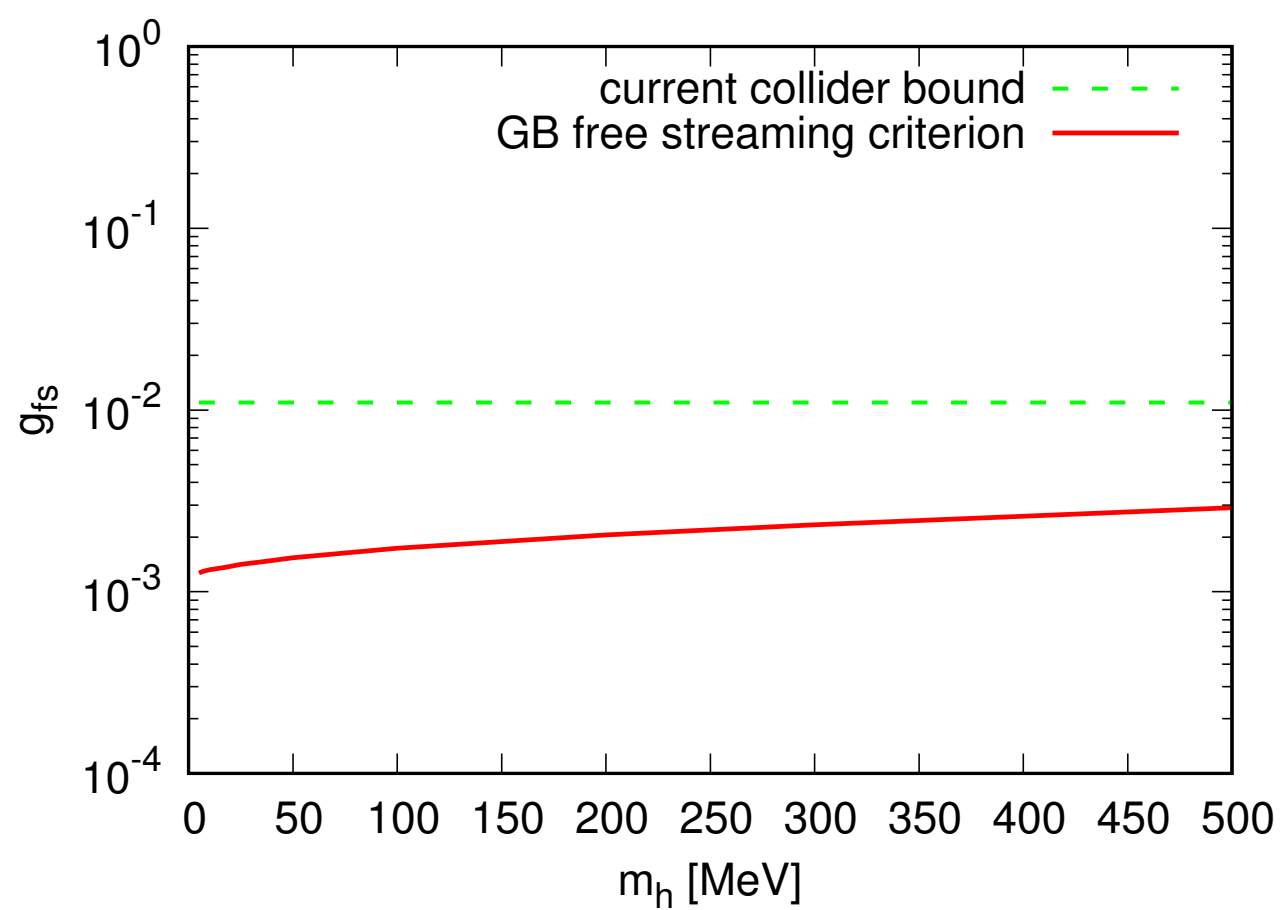

Figure 8. Upper limits on the Higgs portal coupling $g$ for Goldstone boson free-streaming out of the proto-neutron star core, eq. (4.12), for various light Higgs boson mass $m_{h}$ (solid). Also shown is the current collider bound, eq. (2.9) (dashed).

loss argument in other astrophysical objects [37], which we briefly summarise below. As first pointed out in ref. [87], decays of $B$ mesons to $K$ mesons plus missing energy can be an efficient probe of $\mathrm{GeV}$ or sub-GeV scalar dark matter. In refs. $[33,88]$ this consideration has been applied to Weinberg's Higgs portal model. If the light Higgs boson is lighter than $354 \mathrm{MeV}$, the decay of $K$ meson to a pion plus missing energy is a more powerful probe. We follow ref. [33] and use the most stringent constraint on the decay branching ratios,

$$
\mathcal{B}\left(B^{+} \rightarrow K^{+}+h\right)<10^{-5},
$$

by the BaBar experiment [89], and

$$
\mathcal{B}\left(K^{+} \rightarrow \pi^{+}+h\right)<10^{-10},
$$

by the E787 and E949 experiments [90] at the Brookhaven National Laboratory. The former imposes a constraint on the $\varphi-r$ mixing angle (eq. (2.4)) of $\theta<0.0016$ for $m_{h}<m_{B}-m_{K}$, while the latter $\theta<8.7 \cdot 10^{-5}$ for $m_{h}<m_{K}-m_{\pi}=354 \mathrm{MeV}$. Recently, the LHCb Collaboration has published upper limits on the branching fraction $\mathcal{B}\left(B^{+} \rightarrow K^{+} X\right) \times$ $\mathcal{B}\left(X \rightarrow \mu^{+} \mu^{-}\right)$, where $X$ is a hypothetical long-lived scalar particle [91]. The limits at the $95 \%$ confidence level vary between $2 \cdot 10^{-10}$ and $10^{-7}$, for the scalar particle mass in the range $250 \mathrm{MeV}<\mathrm{m}(\mathrm{X})<4700 \mathrm{MeV}$ and lifetime in the range $0.1 \mathrm{ps}<\tau(X)<1000 \mathrm{ps}$. However, since in Weinberg's Higgs portal model we find $\mathcal{B}\left(h \rightarrow \mu^{+} \mu^{-}\right) \lesssim 10^{-12}$, the LHCb upper limits are not applicable. Also shown in figure 9 are exclusion curves derived 


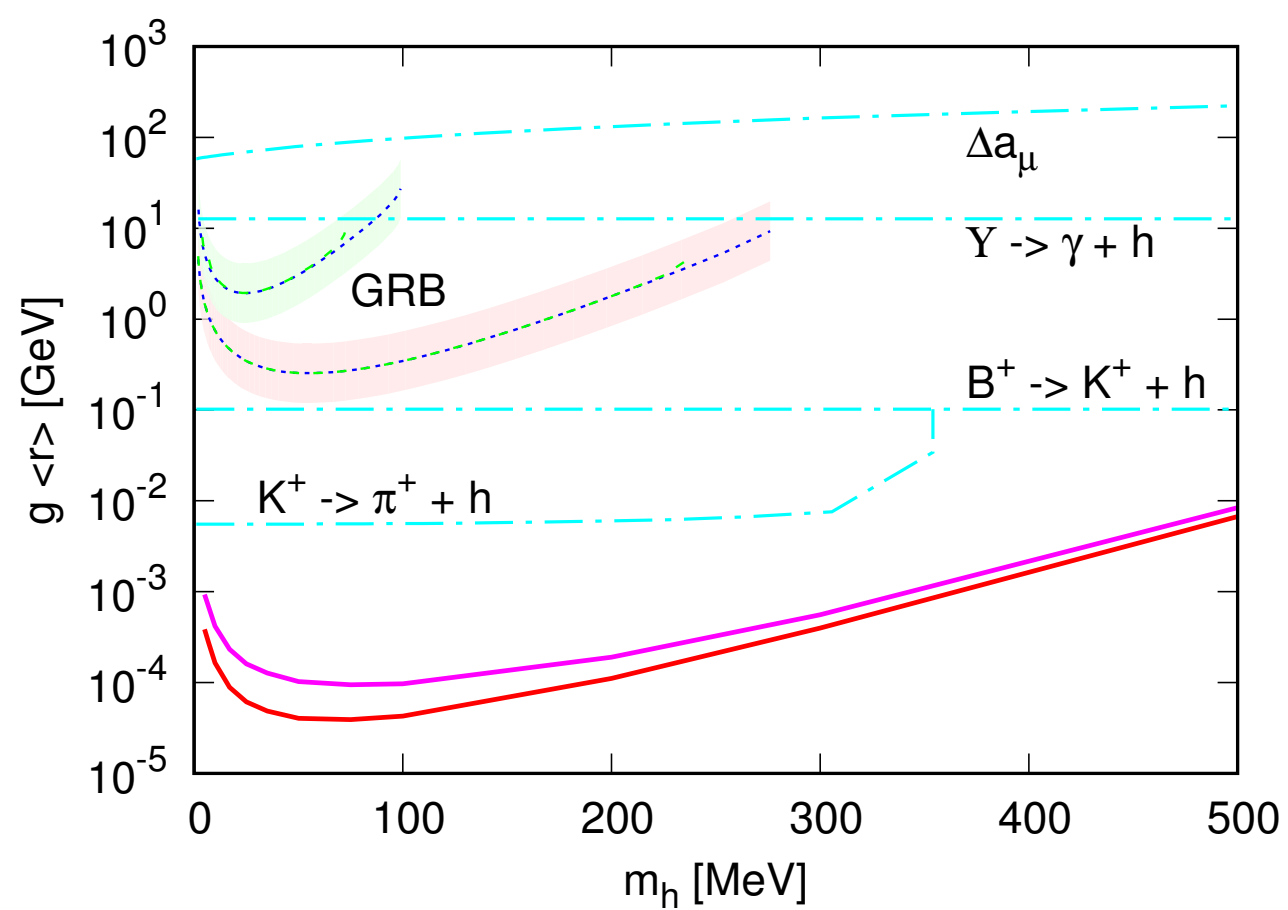

Figure 9. SN 1987A upper limits on $g\langle r\rangle$, the product of the Higgs portal coupling with the vacuum expectation value of the radial field $r$, for various light Higgs boson mass $m_{h}$ (solid lines). The upper solid curve is derived by using the SP07 global fits for the nucleon-nucleon elastic scattering cross section in the energy loss rate calculation, and the lower one by using the one-pion exchange (OPE) approximation. Also shown are the upper limits set by laboratory experiments (dash-dotted lines, from top to bottom), such as the muon anomalous magnetic moment $\Delta a_{\mu}$, radiative Upsilon decays $\Upsilon(n s) \rightarrow \gamma+h, B$ meson invisible decay $B^{+} \rightarrow K^{+}+h$, as well as $K$ meson invisible decay $K^{+} \rightarrow \pi^{+}+h$. The dotted and the dashed lines labelled "GRB" are the upper limits we derived in ref. [37] by invoking the energy loss argument on the initial fireballs of gamma-ray bursts. Two GRB initial fireball temperatures values $T_{0}=18 \mathrm{MeV}$ (lower) and $8 \mathrm{MeV}$ (upper) are assumed, and the Higgs portal coupling $g$ is taken to saturate the current collider bound (dotted) and at future collider sensitivities (dashed). The uncertainties in these GRB upper limits resulting from the error in the GRB energy loss argument, eq. (5.4), are indicated by the filled regions.

using radiative Upsilon decays, $\mathcal{B}(\Upsilon(n S) \rightarrow \gamma+h)<3 \cdot 10^{-6}$, as well as muon anomalous magnetic moment, $\Delta a_{\mu}=288 \cdot 10^{-11}$. Neither of them is useful for constraining $g\langle r\rangle$.

In our previous work [37] we have derived constraints using gamma-ray bursts (GRB) observations. Due to resonance effects, Goldstone boson pairs can be rapidly produced by electron-positron annihilation process in the initial fireballs of the GRBs. On the other hand, the mean free path of the Goldstone bosons is larger than the size of the GRB initial fireballs, so they are not coupled to the GRB's relativistic flow and can lead to significant energy loss. Our GRB energy loss criterion is

$$
Q_{e^{+} e^{-} \rightarrow \alpha \alpha} \Delta t^{\prime} \approx Q_{e^{+} e^{-} \rightarrow \alpha \alpha} \frac{1}{\Gamma_{0}} \frac{\Delta R_{0}}{\beta_{0}} \gtrsim \frac{\mathcal{E}}{\Gamma_{0} V_{0}},
$$

where $\Delta t^{\prime}$ is the time duration in the fireball comoving frame for the GRB initial fireball to expand from the initial radius $R_{0}$ to $R_{0}+\Delta R_{0}$, and $V_{0}$ is the initial fireball volume. 
We used generic values for the GRB initial fireballs, such as total energy $\mathcal{E}=10^{52} \mathrm{erg}$, temperature $T_{0}=18 \mathrm{MeV}$ as well as $8 \mathrm{MeV}$, radius $R_{0}=10^{6.5} \mathrm{~cm}$, wind velocity $\beta_{0}=1 / \sqrt{3}$, and the Lorentz factor is $\Gamma_{0}=1 / \sqrt{1-\beta_{0}^{2}}$. In fact, the GRB bounds on $g\langle r\rangle$ have a slight dependence on the Higgs portal coupling $g$, which becomes visible when the light Higgs boson decay braching ratio to a pair of SM fermions, $\Gamma_{h \rightarrow f \bar{f}}$, is no longer negligible compared to that to a pair of Goldstone bosons, $\Gamma_{h \rightarrow \alpha \alpha}$. We therefore considered $g=0.011$ saturating the current collider bounds, as well as $g=0.0015$ which might be probed by future collider experiments. The region bounded by the two GRB exclusion curves, including the filled regions around them, represents the parameter space in Weinberg's Higgs portal model that can be probed by GRB physics. The GRB bounds are subject to large uncertainties, and are much weaker than the SN 1987A constraints. However, they are competitive to current laboratory constraints in the mass range of $m_{h} / T_{0} \lesssim 10-15$. We conclude here that Weinberg's Higgs portal model is another example to elucidate that high-energy astrophysical objects are excellent laboratory for particle physics.

In the extended version of Weinberg's Higgs portal model, the spin-independent WIMP-nucleon elastic scattering cross section is (following the definition given in e.g. ref. [92])

$$
\sigma_{\chi N}^{\mathrm{SI}}=\frac{4}{\pi}\left(\frac{1}{\sqrt{2}}\right)^{2} \mu_{\chi N}^{2}\left(\frac{f_{\chi} g\langle r\rangle f_{N} m_{N}}{m_{H}^{2} m_{h}^{2}}\right)^{2} .
$$

Here $\mu_{\chi N}=M_{\chi} m_{N} /\left(M_{\chi}+m_{N}\right)$ is the WIMP-nucleon reduced mass. Latest exclusion limits published by the dark matter direct search experiments LUX [34], PANDA-X [93], and XENON1T [35] are translated into constraints on the parameter combination $f_{\chi} g\langle r\rangle / m_{h}^{2}$ for WIMP mass $M_{\chi}$ ranging from $6 \mathrm{GeV}$ to $1 \mathrm{TeV}$. In order to make a comparison to the $\mathrm{SN}$ 1987A and laboratory constraints, the WIMP coupling is fixed by requiring the relic density to be $\Omega_{\chi} h^{2} \simeq 0.11$, which yields $f_{\chi} \approx 0.02 \sqrt{M_{\chi}}$ [44]. The DM constraint was first derived in ref. [33], and here in figure 10 is shown for some representative values of WIMP mass $M_{\chi}=6,10$ and $100 \mathrm{GeV}$. Note that it does not become more stringent for larger WIMP masses, because the experimental limits on $\sigma_{\chi N}^{\mathrm{SI}}$ also scales approximately with $\sqrt{M_{\chi}}$ for $M_{\chi} \geq 100 \mathrm{GeV}$. We conclude that SN 1987A constraints are comparable to bounds from DM direct search results for $M_{\chi} \lesssim 10 \mathrm{GeV}$, while DM bounds for $M_{\chi} \gtrsim 100 \mathrm{GeV}$ are the strongest bounds among all on the extended version of Weinberg's Higgs portal model.

\section{Summary}

Weinberg's Higgs portal model is another example to elucidate that high-energy astrophysical objects such as the supernovae and gamma-ray bursts are excellent laboratory for particle physics. In this model, massless Goldstone bosons arising from the spontaneous breaking of a $U(1)$ symmetry play the role of the dark radiation. The model was also extended to include a Majorana fermion of mass in the $\mathrm{GeV}$ to $\mathrm{TeV}$ range as the dark matter candidate. Both particle species couple to the Standard Model fields solely through the SM Higgs boson.

Goldstone boson production in the proto-neutron star core is dominated by the emission of a real light Higgs boson in nuclear bremsstrahlung processes and its subsequent decay. The SN 1987A constraint on Weinberg's Higgs portal model is obtained by finding 


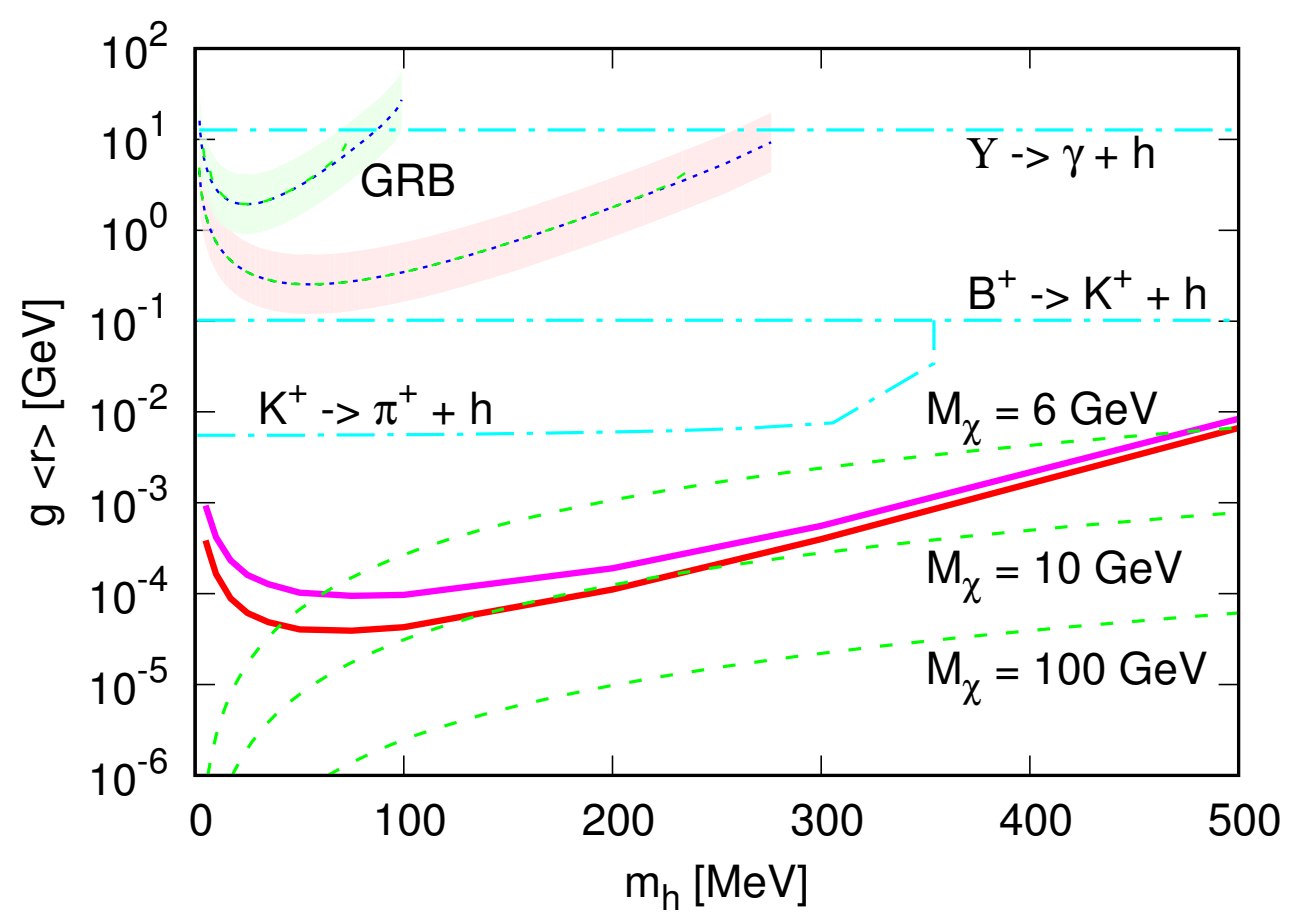

Figure 10. Same as figure 9, here including the upper limits set by the dark matter direct search experiment LUX, for WIMP mass $M_{\chi}=6,10$ and $100 \mathrm{GeV}$ (dashed lines, from top to bottom).

the parameter regions for the Higgs portal coupling $g$, and the vacuum expectation value of the light Higgs boson $\langle r\rangle$, for each light Higgs boson mass $m_{h}$, such that the energy loss rate due to Goldstone boson emission satisfy the Raffelt criterion. In order to invoke this criterion, the Higgs portal coupling $g$ is required to be smaller than the current collider bound inferred from the SM Higgs invisible decay, so that the Goldstone bosons are not trapped inside the proto-neutron star core.

To make conservative estimates for the energy loss rate, in this work we take the nondegenerate (ND) limit. For simplicity we assume the neutron fraction is $X_{n}=1$. We found that using the one-pion exchange (OPE) approximation and the SP07 global fits for the $p p$ elastic cross section results only in a factor of 2.6 difference for $10 \mathrm{MeV} \lesssim m_{h} \lesssim 50 \mathrm{MeV}$, and a factor of 1.4 for $m_{h}>300 \mathrm{MeV}$. The SN 1987A constraints surpass those set by laboratory experiments or by energy loss arguments in other astrophysical objects, even with nuclear uncertainties taken into account. Numerical simulations of supernova cooling with axion or Kaluza-Klein graviton emission included have demonstrated the reliability of the analytical energy loss estimate by Raffelt. Therefore we do not expect significant change in our supernova bounds if simulation for Goldstone boson emission is to be performed. In the extended version of Weinberg's Higgs portal model, latest exclusion limits published by the dark matter direct search experiments LUX, PANDA-X, and XENON1T are translated into constraints on the parameter combination $f_{\chi} g\langle r\rangle / m_{h}^{2}$ for WIMP mass $M_{\chi}$ ranging from $6 \mathrm{GeV}$ to $1 \mathrm{TeV}$. Fixing the WIMP coupling $f_{\chi}$ with the measured dark matter relic density, we found that SN 1987A constraints are comparable to bounds from DM direct search results for WIMP mass $M_{\chi} \lesssim 10 \mathrm{GeV}$, while DM bounds for $M_{\chi} \gtrsim 100 \mathrm{GeV}$ are the strongest bounds among all. 


\section{Acknowledgments}

We thank Xian-Wei Kang, Meng-Ru Wu, Tobias Fischer, and Jusak Tandean for the helpful discussions. This work was supported in part by the Ministry of Science and Technology of Taiwan under the Grant No. 104-2112-M-001-039-MY3.

Open Access. This article is distributed under the terms of the Creative Commons Attribution License (CC-BY 4.0), which permits any use, distribution and reproduction in any medium, provided the original author(s) and source are credited.

\section{References}

[1] G.G. Raffelt, Stars as laboratories for fundamental physics: The astrophysics of neutrinos, axions, and other weakly interacting particles, University of Chicago Press, Chicago, U.S.A. (1996), pg. 664 [INSPIRE].

[2] T. Fischer, S.C. Whitehouse, A. Mezzacappa, F.K. Thielemann and M. Liebendorfer, Protoneutron star evolution and the neutrino driven wind in general relativistic neutrino radiation hydrodynamics simulations, Astron. Astrophys. 517 (2010) A80 [arXiv:0908.1871] [INSPIRE].

[3] B. Müller and H.-T. Janka, A New Multi-Dimensional General Relativistic Neutrino Hydrodynamics Code for Core-Collapse Supernovae IV. The Neutrino Signal, Astrophys. J. 788 (2014) 82 [arXiv:1402.3415] [INSPIRE].

[4] G. Camelio, A. Lovato, L. Gualtieri, O. Benhar, J.A. Pons and V. Ferrari, Evolution of a proto-neutron star with a nuclear many-body equation of state: neutrino luminosity and gravitational wave frequencies, arXiv:1704.01923 [INSPIRE].

[5] M. Prakash, I. Bombaci, M. Prakash, P.J. Ellis, J.M. Lattimer and R. Knorren, Composition and structure of protoneutron stars, Phys. Rept. 280 (1997) 1 [nucl-th/9603042] [INSPIRE].

[6] J.A. Pons, S. Reddy, M. Prakash, J.M. Lattimer and J.A. Miralles, Evolution of protoneutron stars, Astrophys. J. 513 (1999) 780 [astro-ph/9807040] [INSPIRE].

[7] O.E. Nicotra, M. Baldo, G.F. Burgio and H.J. Schulze, Protoneutron stars within the Brueckner-Bethe-Goldstone theory, Astron. Astrophys. 451 (2006) 213 [nucl-th/0506066] [INSPIRE].

[8] H.T. Janka, Neutrino Emission from Supernovae, arXiv:1702.08713 [INSPIRE].

[9] G. Raffelt and D. Seckel, Bounds on Exotic Particle Interactions from SN 1987a, Phys. Rev. Lett. 60 (1988) 1793 [INSPIRE].

[10] M.S. Turner, Axions from SN 1987a, Phys. Rev. Lett. 60 (1988) 1797 [InSPIRE].

[11] R. Mayle, J.R. Wilson, J.R. Ellis, K.A. Olive, D.N. Schramm and G. Steigman, Constraints on Axions from SN 1987a, Phys. Lett. B 203 (1988) 188 [INSPIRE].

[12] R.P. Brinkmann and M.S. Turner, Numerical Rates for Nucleon-Nucleon Axion Bremsstrahlung, Phys. Rev. D 38 (1988) 2338 [InSPIRE].

[13] H.-T. Janka, W. Keil, G. Raffelt and D. Seckel, Nucleon spin fluctuations and the supernova emission of neutrinos and axions, Phys. Rev. Lett. 76 (1996) 2621 [astro-ph/9507023] [INSPIRE]. 
[14] C. Hanhart, D.R. Phillips, S. Reddy and M.J. Savage, Extra dimensions, SN1987a and nucleon-nucleon scattering data, Nucl. Phys. B 595 (2001) 335 [nucl-th/0007016] [INSPIRE].

[15] C. Hanhart, J.A. Pons, D.R. Phillips and S. Reddy, The Likelihood of GODs' existence: Improving the SN1987a constraint on the size of large compact dimensions, Phys. Lett. B 509 (2001) 1 [astro-ph/0102063] [INSPIRE].

[16] S. Hannestad and G.G. Raffelt, Supernova and neutron star limits on large extra dimensions reexamined, Phys. Rev. D 67 (2003) 125008 [Erratum ibid. D 69 (2004) 029901] [hep-ph/0304029] [INSPIRE].

[17] S. Hannestad, G. Raffelt and Y.Y.Y. Wong, Unparticle constraints from SN 1987A, Phys. Rev. D 76 (2007) 121701 [arXiv:0708.1404] [INSPIRE].

[18] A. Freitas and D. Wyler, Astro Unparticle Physics, JHEP 12 (2007) 033 [arXiv:0708.4339] [INSPIRE].

[19] J.H. Chang, R. Essig and S.D. McDermott, Revisiting Supernova 1987A Constraints on Dark Photons, JHEP 01 (2017) 107 [arXiv: 1611.03864] [INSPIRE].

[20] E. Hardy and R. Lasenby, Stellar cooling bounds on new light particles: plasma mixing effects, JHEP 02 (2017) 033 [arXiv: 1611.05852] [INSPIRE].

[21] A. Guha, S.J. and P.K. Das, $q$-deformed statistics and the role of light fermionic dark matter in SN1987A cooling, Phys. Rev. D 95 (2017) 015001 [arXiv:1509.05901] [INSPIRE].

[22] N. Ishizuka and M. Yoshimura, Axion and Dilaton Emissivity From Nascent Neutron Stars, Prog. Theor. Phys. 84 (1990) 233 [INSPIRE].

[23] D. Arndt and P.J. Fox, Saxion emission from SN1987A, JHEP 02 (2003) 036 [hep-ph/0207098] [INSPIRE].

[24] W. Keil, H.-T. Janka, D.N. Schramm, G. Sigl, M.S. Turner and J.R. Ellis, A Fresh look at axions and SN-1987A, Phys. Rev. D 56 (1997) 2419 [astro-ph/9612222] [INSPIRE].

[25] T. Fischer, S. Chakraborty, M. Giannotti, A. Mirizzi, A. Payez and A. Ringwald, Probing axions with the neutrino signal from the next galactic supernova, Phys. Rev. D 94 (2016) 085012 [arXiv: 1605.08780] [INSPIRE].

[26] G.G. Raffelt, Astrophysical methods to constrain axions and other novel particle phenomena, Phys. Rept. 198 (1990) 1 [INSPIRE].

[27] G.G. Raffelt, Astrophysical axion bounds, Lect. Notes Phys. 741 (2008) 51 [hep-ph/0611350] [INSPIRE].

[28] S. Weinberg, Goldstone Bosons as Fractional Cosmic Neutrinos, Phys. Rev. Lett. 110 (2013) 241301 [arXiv: 1305.1971] [INSPIRE].

[29] A.G. Riess et al., A 2.4\% Determination of the Local Value of the Hubble Constant, Astrophys. J. 826 (2016) 56 [arXiv:1604.01424] [INSPIRE].

[30] A. Heavens et al., No evidence for extensions to the standard cosmological model, arXiv: 1704.03467 [INSPIRE].

[31] K.-W. Ng, H. Tu and T.-C. Yuan, Dark photons as fractional cosmic neutrino masquerader, JCAP 09 (2014) 035 [arXiv: 1406.1993] [INSPIRE].

[32] K. Cheung, W.-Y. Keung and T.-C. Yuan, Collider Signatures of Goldstone Bosons, Phys. Rev. D 89 (2014) 015007 [arXiv: 1308.4235] [INSPIRE]. 
[33] L.A. Anchordoqui et al., Weinberg's Higgs portal confronting recent LUX and LHC results together with upper limits on $B^{+}$and $K^{+}$decay into invisibles, Phys. Rev. D 89 (2014) 083513 [arXiv: 1312.2547] [INSPIRE].

[34] LUX collaboration, D.S. Akerib et al., Results from a search for dark matter in the complete LUX exposure, Phys. Rev. Lett. 118 (2017) 021303 [arXiv: 1608.07648] [INSPIRE].

[35] XENON collaboration, E. Aprile et al., First Dark Matter Search Results from the XENON1T Experiment, arXiv:1705.06655 [INSPIRE].

[36] W.-Y. Keung, K.-W. Ng, H. Tu and T.-C. Yuan, Supernova bounds on Weinberg's Goldstone bosons, Phys. Rev. D 90 (2014) 075014 [arXiv:1312.3488] [InSPIRE].

[37] H. Tu and K.-W. Ng, Effects of Goldstone Bosons on Gamma-Ray Bursts, JCAP 03 (2016) 037 [arXiv: 1512.05165] [INSPIRE].

[38] M. Drees and M. Nojiri, Neutralino-nucleon scattering revisited, Phys. Rev. D 48 (1993) 3483 [hep-ph/9307208] [INSPIRE].

[39] G. Jungman, M. Kamionkowski and K. Griest, Supersymmetric dark matter, Phys. Rept. 267 (1996) 195 [hep-ph/9506380] [INSPIRE].

[40] J. Hisano, K. Ishiwata, N. Nagata and T. Takesako, Direct Detection of Electroweak-Interacting Dark Matter, JHEP 07 (2011) 005 [arXiv:1104.0228] [INSPIRE].

[41] H.-Y. Cheng and C.-W. Chiang, Revisiting Scalar and Pseudoscalar Couplings with Nucleons, JHEP 07 (2012) 009 [arXiv: 1202.1292] [INSPIRE].

[42] J.M. Cline, K. Kainulainen, P. Scott and C. Weniger, Update on scalar singlet dark matter, Phys. Rev. D 88 (2013) 055025 [Erratum ibid. D 92 (2015) 039906] [arXiv:1306.4710] [INSPIRE].

[43] X.-G. He and J. Tandean, Low-Mass Dark-Matter Hint from CDMS II, Higgs Boson at the LHC and Darkon Models, Phys. Rev. D 88 (2013) 013020 [arXiv:1304.6058] [inSPIRE].

[44] L.A. Anchordoqui and B.J. Vlcek, W-WIMP Annihilation as a Source of the Fermi Bubbles, Phys. Rev. D 88 (2013) 043513 [arXiv: 1305.4625] [INSPIRE].

[45] P. Bechtle, S. Heinemeyer, O. Stål, T. Stefaniak and G. Weiglein, Probing the Standard Model with Higgs signal rates from the Tevatron, the LHC and a future ILC, JHEP 11 (2014) 039 [arXiv: 1403.1582] [INSPIRE].

[46] M. Taketani, S. Nakamura and M. Sasaki, On the Method of the Theory of Nuclear Forces, Prog. Theor. Phys. 6 (1951) 581.

[47] D.R. Entem, R. Machleidt and Y. Nosyk, Consistent, high-quality two-nucleon potentials up to fifth order of the chiral expansion, arXiv:1703.05454 [INSPIRE].

[48] D.R. Entem, N. Kaiser, R. Machleidt and Y. Nosyk, Dominant contributions to the nucleon-nucleon interaction at sixth order of chiral perturbation theory, Phys. Rev. C 92 (2015) 064001 [arXiv: 1505.03562] [INSPIRE].

[49] R. Machleidt and I. Slaus, The nucleon-nucleon interaction: Topical review, J. Phys. G 27 (2001) R69 [nucl-th/0101056] [INSPIRE].

[50] M. Naghdi, Nucleon-nucleon interaction: A typical/concise review, Phys. Part. Nucl. 45 (2014) 924 [nucl-th/0702078] [INSPIRE].

[51] R. Machleidt and D.R. Entem, Chiral effective field theory and nuclear forces, Phys. Rept. $\mathbf{5 0 3}$ (2011) 1 [arXiv:1105.2919] [INSPIRE]. 
[52] R. Machleidt, Chiral Symmetry and the Nucleon-Nucleon Interaction, Symmetry 8 (2016) 26 [INSPIRE].

[53] S. Bacca, K. Hally, C.J. Pethick and A. Schwenk, Chiral effective field theory calculations of neutrino processes in dense matter, Phys. Rev. C 80 (2009) 032802 [arXiv:0812.0102] [INSPIRE].

[54] S. Bacca, R. Sharma and A. Schwenk, Emission of neutrino-antineutrino pairs by hadronic bremsstrahlung processes, EPJ Web Conf. 117 (2016) 02003 [arXiv: 1509.08151] [INSPIRE].

[55] B.L. Friman and O.V. Maxwell, Neutron Star Neutrino Emissivities, Astrophys. J. 232 (1979) 541 [INSPIRE].

[56] S. Hannestad and G. Raffelt, Supernova neutrino opacity from nucleon-nucleon Bremsstrahlung and related processes, Astrophys. J. 507 (1998) 339 [astro-ph/9711132] [INSPIRE].

[57] A. Bartl, R. Bollig, H.-T. Janka and A. Schwenk, Impact of Nucleon-Nucleon Bremsstrahlung Rates Beyond One-Pion Exchange, Phys. Rev. D 94 (2016) 083009 [arXiv:1608.05037] [INSPIRE].

[58] A. Bartl, C.J. Pethick and A. Schwenk, Supernova matter at subnuclear densities as a resonant Fermi gas: Enhancement of neutrino rates, Phys. Rev. Lett. 113 (2014) 081101 [arXiv: 1403.4114] [INSPIRE].

[59] C. Hanhart, D.R. Phillips and S. Reddy, Neutrino and axion emissivities of neutron stars from nucleon-nucleon scattering data, Phys. Lett. B 499 (2001) 9 [astro-ph/0003445] [INSPIRE].

[60] V. Limkaisang et al., Phase-shift analysis of pp scattering at $T_{L}=25-500 \mathrm{MeV}$, Prog. Theor. Phys. 105 (2001) 233 [INSPIRE].

[61] V.A. Babenko and N.M. Petrov, Study of the Pion-Nucleon Coupling Constant Charge Dependence on the Basis of the Low-Energy Data on Nucleon-Nucleon Interaction, arXiv: 1604.02912 [INSPIRE].

[62] G. Martinez-Pinedo, T. Fischer, A. Lohs and L. Huther, Charged-current weak interaction processes in hot and dense matter and its impact on the spectra of neutrinos emitted from proto-neutron star cooling, Phys. Rev. Lett. 109 (2012) 251104 [arXiv:1205.2793] [INSPIRE].

[63] M. Baldo and G.F. Burgio, The nuclear symmetry energy, Prog. Part. Nucl. Phys. 91 (2016) 203 [arXiv: 1606.08838] [INSPIRE].

[64] W. Trautmann, M.D. Cozma and P. Russotto, Symmetry energy and density, PoS (Bormio2016) 036 [arXiv: 1610.03650] [INSPIRE].

[65] S. Bacca, K. Hally, M. Liebendorfer, A. Perego, C.J. Pethick and A. Schwenk, Neutrino processes in partially degenerate neutron matter, Astrophys. J. 758 (2012) 34 [arXiv:1112.5185] [INSPIRE].

[66] D. Albers et al., A Precision measurement of pp elastic scattering cross-sections at intermediate energies, Eur. Phys. J. A 22 (2004) 125 [nucl-ex/0403045] [InSPIRE].

[67] C. Wilkin, The legacy of the hadron physics programme at COSY, EPJ Web Conf. 130 (2016) 01007 [INSPIRE].

[68] R.A. Arndt, I.I. Strakovsky and R.L. Workman, Nucleon nucleon elastic scattering to $3 \mathrm{GeV}$, Phys. Rev. C 62 (2000) 034005 [nucl-th/0004039] [INSPIRE]. 
[69] R.A. Arndt, W.J. Briscoe, I.I. Strakovsky and R.L. Workman, Updated analysis of NN elastic scattering to 3-GeV, Phys. Rev. C 76 (2007) 025209 [arXiv:0706.2195] [InSPIRE].

[70] http://nn-online.org.

[71] http://gwdac.phys.gwu.edu.

[72] X.W. Kang, Chiral Dynamics and Final State Interactions in Semileptonic B Meson Decay and Antinucleon-Nucleon Scattering, Ph.D. Thesis, University of Bonn (2014), http://hss.ulb.uni-bonn.de/2014/3714/3714.htm.

[73] R.A. Arndt, W.J. Briscoe, A.B. Laptev, I.I. Strakovsky and R.L. Workman, Absolute Total np and pp Cross section Determinations, Nucl. Sci. Eng. 162 (2009) 312 [arXiv:0806.1198] [INSPIRE].

[74] E.S. Konobeevski, S.V. Zuyev, V.I. Kukulin and V.N. Pomerantsev, New estimation for neutron-neutron scattering length: charge symmetry and charge independence breaking revisited, arXiv: 1703.00519 [INSPIRE].

[75] V.G.J. Stoks, R.A.M. Klomp, M.C.M. Rentmeester and J.J. de Swart, Partial wave analaysis of all nucleon-nucleon scattering data below 350-MeV, Phys. Rev. C 48 (1993) 792 [INSPIRE].

[76] F.E. Low, Bremsstrahlung of very low-energy quanta in elementary particle collisions, Phys. Rev. 110 (1958) 974 [INSPIRE].

[77] S.L. Adler and Y. Dothan, Low-energy theorem for the weak axial-vector vertex, Phys. Rev. 151 (1966) 1267 [INSPIRE].

[78] L. Heller, Soft-photon theorem for bremsstrahlung in a potential model, Phys. Rev. 174 (1968) 1580 [INSPIRE].

[79] M.K. Liou, Low-Energy Theorem for Nucleon-Nucleon Bremsstrahlung, Phys. Rev. C 2 (1970) 131 [INSPIRE].

[80] A. Burrows, M.T. Ressell and M.S. Turner, Axions and SN1987A: Axion trapping, Phys. Rev. D 42 (1990) 3297 [INSPIRE].

[81] D.L. Tubbs and D.N. Schramm, Neutrino Opacities at High Temperatures and Densities, Astrophys. J. 201 (1975) 467 [INSPIRE].

[82] S. Guillot, M. Servillat, N.A. Webb and R.E. Rutledge, Measurement of the Radius of Neutron Stars with High S/N Quiescent Low-mass X-ray Binaries in Globular Clusters, Astrophys. J. $\mathbf{7 7 2}$ (2013) 7 [arXiv:1302.0023] [INSPIRE].

[83] C.A. Raithel, F. Ozel and D. Psaltis, Model-Independent Inference of Neutron Star Radii from Moment of Inertia Measurements, Phys. Rev. C 93 (2016) 032801 [arXiv:1603.06594] [INSPIRE].

[84] J.M. Lattimer and M. Prakash, The Equation of State of Hot, Dense Matter and Neutron Stars, Phys. Rept. 621 (2016) 127 [arXiv:1512.07820] [INSPIRE].

[85] M.C. Miller and F.K. Lamb, Observational Constraints on Neutron Star Masses and Radii, Eur. Phys. J. A 52 (2016) 63 [arXiv:1604.03894] [InSPIRE].

[86] M. Baldo, G.F. Burgio, H.J. Schulze and G. Taranto, Nucleon effective masses within the Brueckner-Hartree-Fock theory: Impact on stellar neutrino emission, Phys. Rev. C 89 (2014) 048801 [arXiv: 1404.7031] [INSPIRE]. 
[87] C. Bird, P. Jackson, R.V. Kowalewski and M. Pospelov, Search for dark matter in $b \rightarrow s$ transitions with missing energy, Phys. Rev. Lett. 93 (2004) 201803 [hep-ph/0401195] [INSPIRE].

[88] F.P. Huang, C.S. Li, D.Y. Shao and J. Wang, Phenomenology of an Extended Higgs Portal Inflation Model after Planck 2013, Eur. Phys. J. C 74 (2014) 2990 [arXiv:1307.7458] [INSPIRE].

[89] BaBar collaboration, P. del Amo Sanchez et al., Search for the Rare Decay $B \rightarrow K \nu \bar{\nu}$, Phys. Rev. D 82 (2010) 112002 [arXiv: 1009.1529] [INSPIRE].

[90] BNL-E949 collaboration, A.V. Artamonov et al., Study of the decay $K^{+} \rightarrow \pi^{+} \nu \bar{\nu}$ in the momentum region $140<P_{\pi}<199 \mathrm{MeV} / c$, Phys. Rev. D 79 (2009) 092004 [arXiv: 0903.0030] [INSPIRE].

[91] LHCb collaboration, Search for long-lived scalar particles in $B^{+} \rightarrow K^{+} \chi\left(\mu^{+} \mu^{-}\right)$decays, Phys. Rev. D 95 (2017) 071101 [arXiv:1612.07818] [InSPIRE].

[92] A. Kurylov and M. Kamionkowski, Generalized analysis of weakly interacting massive particle searches, Phys. Rev. D 69 (2004) 063503 [hep-ph/0307185] [INSPIRE].

[93] PandaX-II collaboration, A. Tan et al., Dark Matter Results from First 98.7 Days of Data from the PandaX-II Experiment, Phys. Rev. Lett. 117 (2016) 121303 [arXiv:1607.07400] [INSPIRE]. 